\title{
The limb-brightened jet of M 87 down to the 7 Schwarzschild radii scale ${ }^{\star}$
}

\author{
J.-Y. Kim ${ }^{1}$, T. P. Krichbaum ${ }^{1}$, R.-S. Lu ${ }^{1}$, E. Ros ${ }^{1,2,3}$, U. Bach ${ }^{1}$, M. Bremer ${ }^{4}$, P. de Vicente ${ }^{5}$, \\ M. Lindqvist ${ }^{6}$, and J. A. Zensus ${ }^{1}$

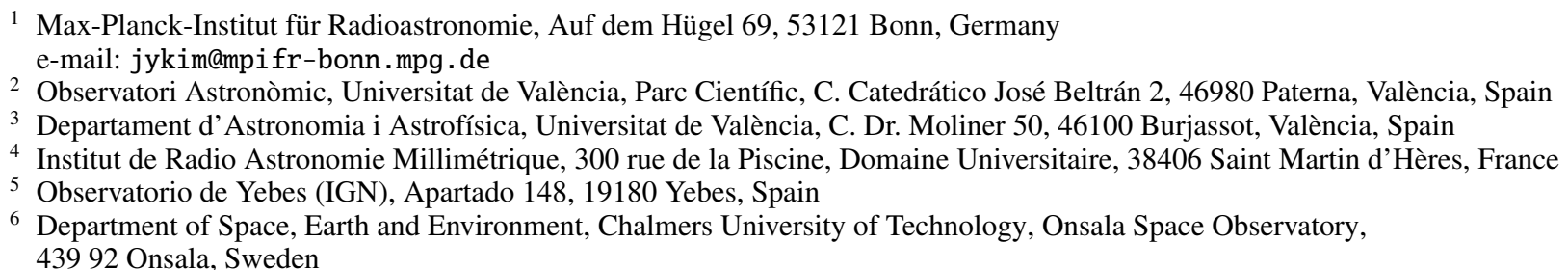

Received 28 February 2018 / Accepted 4 May 2018

\begin{abstract}
Messier 87 (M 87) is one of the nearest radio galaxies with a prominent jet extending from sub-pc to kpc scales. Because of its proximity and the large mass of its central black hole $(\mathrm{BH})$, it is one of the best radio sources for the study of jet formation. We study the physical conditions near the jet base at projected separations from the BH of $\sim 7-100$ Schwarzschild radii $\left(R_{\text {sch }}\right)$. Global mm-VLBI Array (GMVA) observations at $86 \mathrm{GHz}(\lambda=3.5 \mathrm{~mm})$ provide an angular resolution of $\sim 50 \mu$ as, which corresponds to a spatial resolution of only $7 R_{\text {sch }}$ and reach the small spatial scale. We use five GMVA data sets of M 87 obtained from 2004 to 2015 and present new high angular resolution VLBI maps at $86 \mathrm{GHz}$. In particular, we focus on the analysis of the brightness temperature, the jet ridge lines, and the ratio of jet to counter-jet. The imaging reveals a parabolically expanding limb-brightened jet which emanates from a resolved VLBI core of $\sim(8-13) R_{\text {sch }}$ in size. The observed brightness temperature of the core at any epoch is $\sim(1-3) \times 10^{10} \mathrm{~K}$, which is below the equipartition brightness temperature and suggests magnetic energy dominance at the jet base. We estimate the diameter of the jet at its base to be $\sim 5 R_{\text {sch }}$ assuming a self-similar jet structure. This suggests that the sheath of the jet may be anchored in the very inner portion of the accretion disk. The image stacking reveals faint emission at the center of the edge-brightened jet on sub-pc scales. We discuss its physical implication within the context of the spine-sheath structure of the jet.
\end{abstract}

Key words. galaxies: active - galaxies: jets - galaxies: individual: M 87 - techniques: interferometric

\section{Introduction}

The formation and initial acceleration of relativistic jets in active galactic nuclei (AGN) is still one of the open fundamental questions in modern astrophysics. From a theoretical point of view, there is a general agreement that hot accretion flows falling toward central supermassive black holes (SMBHs) can produce collimated streams of highly magnetized plasma, which propagate outwards and are accelerated through general magnetohydrodynamic (GRMHD) processes (see Meier 2012; Yuan \& Narayan 2014 for a review). The two most promising mechanisms for jet launching are the extraction of rotational energy at the spinning $\mathrm{BH}$ ergosphere (e.g., the BZ mechanism; Blandford \& Znajek 1977) and jet launching from a magnetically collimated accretion disk wind (e.g., the BP mechanism; Blandford \& Payne 1982), which are not mutually exclusive (Hardee et al. 2007). Farther out, the magnetic energy stored in the jet is gradually converted into kinetic power as the jet expands and interacts with the jet-ambient medium, which in turn further collimates and accelerates the jet over long

$\star$ The reduced images (FITS files) are only available at the CDS via anonymous ftp to cdsarc.u-strasbg. fr (130.79.128.5) or via http://cdsarc.u-strasbg.fr/viz-bin/qcat?J/A+A/616/A188 distances (McKinney 2006; Komissarov et al. 2007; Lyubarsky 2009; Mościbrodzka et al. 2016).

Observational constraints on theoretical models are best obtained from imaging of inner jet regions through high-resolution VLBI imaging. In this regard and owing to its proximity, the nearby giant elliptical galaxy Messier 87 (M 87) (1228+126, $3 \mathrm{C} 274$, Virgo A) is an ideal laboratory for the study of jet launching and the coupling of the jet to the accretion flow and the central BH. M 87 is at a distance of 16.7 Mpc (Bird et al. 2010), which yields an angular to linear conversion scale of 1 mas $\sim 0.08 \mathrm{pc}$. Adopting the mass of the SMBH $M_{\mathrm{BH}}=6.1 \times 10^{9} M_{\odot}$ (using the mass from Gebhardt et al. 2011 adjusted to the distance above), an angular scale of 1 mas corresponds to a spatial scale of $\approx 140 R_{\mathrm{sch}}{ }^{1}$. At $86 \mathrm{GHz}$ and with a $50 \mu$ as VLBI observing beam, it is therefore possible to image the jet base with a spatial resolution of $7 R_{\text {sch }}$.

The structure and dynamics of the M 87 jet have been extensively studied in previous VLBI observations. Junor et al. (1999)

1 There is a factor of 2 uncertainty in the assumed $\mathrm{BH}$ mass for $\mathrm{M} 87$, depending on the $\mathrm{BH}$ mass determination method (compare Walsh et al. 2013 with Gebhardt et al. 2011). In this paper, we use $M_{\mathrm{BH}}=6.1 \times 10^{9} M_{\mathrm{BH}}$ for consistency with recent VLBI studies of the M 87 jet. 
Table 1. Summary of the $86 \mathrm{GHz}$ GMVA observations of M 87 .

\begin{tabular}{|c|c|c|c|c|c|c|}
\hline $\begin{array}{c}\text { Date } \\
(\text { yyyy/mm/dd) } \\
(1)\end{array}$ & $\begin{array}{c}\text { Stations } \\
\text { (2) }\end{array}$ & $\begin{array}{c}\text { Beam } \\
\left(b_{\text {min }} \times b_{\text {maj }}, b_{\mathrm{PA}}\right) \\
(3)\end{array}$ & $\begin{array}{l}\text { Pol } \\
\text { (4) }\end{array}$ & $\begin{array}{c}\Delta v \\
(\mathrm{MHz}) \\
(5)\end{array}$ & $\begin{array}{c}\text { Peak } \\
\left(\mathrm{mJy} \mathrm{beam}^{-1}\right) \\
(6)\end{array}$ & $\begin{array}{c}\mathrm{rms} \\
\left(\mathrm{mJy} \mathrm{beam}^{-1}\right) \\
(7)\end{array}$ \\
\hline 2004/04/19 & $\mathrm{EB}, \mathrm{ON}, \mathrm{PV}, \mathrm{VLBA}^{a}$ & $71 \times 267,-9.14$ & $\mathrm{~L}$ & 128 & 411 & 0.26 \\
\hline $2005 / 10 / 15$ & $\mathrm{~EB}, \mathrm{ON}, \mathrm{PV}, \mathrm{VLBA}$ & $61 \times 214,-5.04$ & $\mathrm{~L}$ & 128 & 408 & 0.27 \\
\hline $2009 / 05 / 09-10^{b}$ & $\mathrm{~EB}, \mathrm{ON}, \mathrm{PB}(6), \mathrm{VLBA}$ & $72 \times 272,-10.3$ & $\mathrm{~L}$ & 128 & 758 & 0.24 \\
\hline $2014 / 02 / 26$ & GBT, VLBA & $116 \times 307,-9.0$ & L\&R & 512 & 496 & 0.14 \\
\hline $2015 / 05 / 16$ & $\mathrm{~EB}, \mathrm{ON}, \mathrm{PV}, \mathrm{PB}(5), \mathrm{GBT}, \mathrm{VLBA}$ & $59 \times 273,-6.88$ & L\&R & $512^{c}$ & 351 & 0.16 \\
\hline
\end{tabular}

Notes. Station abbreviations are: EB - Effelsberg, ON - Onsala, PV - the IRAM 30 m telescope at Pico Veleta, PB - the phased Plateau de Bure interferometer (number of the phased antennas in brackets), GBT - the Green Bank Telescope, and VLBA - 8 VLBA stations equipped with 3 mm receiver (without Hancock and Saint Croix). The synthetic beam sizes are for natural weighting; angle denotes the position angle of the major axis. Columns denote: (1) observing date; (2) participating stations; (3) beam sizes: major axis and minor axis (in $\mu$ as), and position angle (in $\mathrm{deg}$ ); (4) receiver polarization ( $\mathrm{L}=\mathrm{LCP} ; \mathrm{R}=\mathrm{RCP}$ ); (5) total bandwidth; $(6,7)$ peak and rms noise level in the resulting images. ${ }^{(a)}$ Brewster was not available; ${ }^{(b)}$ Observations in 2009 were conducted over two consecutive days; ${ }^{(c)} \mathrm{PB}$ observed with a reduced bandwidth of $256 \mathrm{MHz}(1 \mathrm{Gbps}$ recording rate).

discovered a large apparent jet opening angle of $\sim 60^{\circ}$ on subpc scales, which is much wider than the opening angle on the kpc-scale jet $\left(\sim 10^{\circ}\right)$. This suggests ongoing collimation of the jet flow between sub-pc and pc-scales. An edge-brightened jet morphology and a faint counter-jet were seen in multi-epoch Very Long Baseline Array (VLBA) observations at 15 and $43 \mathrm{GHz}$ (Kovalev et al. 2007; Ly et al. 2007). The study of the jet collimation profile by Asada \& Nakamura (2012) and Hada et al. (2013) have revealed a parabolic jet shape up to the Bondi radius, and free conical expansion beyond. The variation in the jet expansion profile could be explained through a variation in the external pressure (Komissarov et al. 2007; Lyubarsky 2009).

In M 87 the jet kinematics is complex. Several distinct, moving emission features suggest a systematic acceleration from subluminal motion near the VLBI core $(\lessgtr 1 c$ at $\lesssim 0.1 \mathrm{pc}$ projected core separation) to superluminal velocities on $\mathrm{pc}$ and also on kpc scales (up to $6 c$; Biretta et al. 1999; Cheung et al. 2007; Kovalev et al. 2007; Walker et al. 2008; Asada et al. 2014; Hada et al. 2016; Mertens et al. 2016). However, subluminal motion is also seen at these larger core distances. This has been interpreted as plasma instabilities and moving patterns within the jet (Mertens et al. 2016).

We note that Global VLBI observations at $230 \mathrm{GHz}$ with the Event Horizon Telescope (EHT; e.g., Doeleman et al. 2012) measure the size of the VLBI components, which are associated with the jet launching region near the event horizon of the central BH. Krichbaum et al. (2014) has reported a tentative size of the VLBI core of $\leq 3.5 R_{\text {sch }}$ from a three-component Gaussian model fitting to more recent EHT data. However, the still limited $(u, v)$-coverage of the previous EHT observations does not yet allow imaging of M 87 with high fidelity at this frequency.

With these restrictions in mind, Global Millimeter VLBI Array (GMVA) observations (e.g., Martí-Vidal et al. 2012; Krichbaum et al. 2014) at $86 \mathrm{GHz}$ play a crucial role in further tracing the M87 jet towards its origin. With the GMVA, it is possible to directly compare the structure of the innermost jet of M87 observed at the above-mentioned spatial resolution of $7 R_{\text {sch }}$ with GRMHD simulations of the jet formation region (e.g., McKinney 2006; Tchekhovskoy et al. 2011; Mościbrodzka et al. 2016). GMVA $86 \mathrm{GHz}$ observations are also crucial in order to bridge the gap between the larger-scale jet morphology seen at observing frequencies $v \leq 43 \mathrm{GHz}$ and the event horizon-scale structures expected to be observed at $v \geq 230 \mathrm{GHz}$ by future EHT observations.
The GMVA observations of M 87 since 2004 have shown an edge-brightened core-jet structure tracing the jet at up to $\sim 3$ mas separation from the VLBI core (Krichbaum et al. 2006, 2014; Kim et al. 2016). These early observations have been confirmed and complemented by follow-up studies with the VLBA and the Green Bank Telescope (GBT; Hada et al. 2016) which have resolution that is a factor of $\sim 2$ lower. These data also show a limbbrightened jet structure which allow a quantitative study of the jet collimation profile down to 0.1 mas core separation. In the GMVA observations of May 2015 the number of participating telescopes was further increased, including the GBT, the IRAM $30 \mathrm{~m}$ telescope, and the phased Plateau de Bure interferometer. This led to enhanced $(u, v)$-coverage and imaging sensitivity and provided a new $86 \mathrm{GHz}$ VLBI image of M 87 which we present in this paper.

In Sect. 2 we summarize the observations and data reduction scheme. We present the main results and the analysis in Sect. 3. In Sect. 4 we discuss the physical meaning of our findings in a broader context. In Sect. 5 we summarize the results.

Throughout the paper we assume a $\Lambda \mathrm{CDM}$ cosmology with cosmological constants $H_{0}=71 \mathrm{~km} \mathrm{~s}^{-1} \mathrm{Mpc}^{-1}, \Omega_{m}=0.27$, and $\Omega_{\Lambda}=0.73$ (Komatsu et al. 2011). Unless specifically mentioned, the length scales in $R_{\text {sch }}$ and parsec(s) refer to deprojected distances along the jet axis based on the jet viewing angle of $18^{\circ}$ determined from the kinematics of the jet (Mertens et al. 2016).

\section{GMVA observations and data reduction}

M 87 has been observed by the GMVA during 2002 and 2015 (Krichbaum et al. 2006, 2014; Kim et al. 2016). In all observations M 87 and the calibrator 3C 273 were observed in full $(u, v)$-tracks, with up to $15 \mathrm{~h}$ mutual visibility between the stations. Here we focus on the data obtained after 2004 which allow reliable imaging of the core and the limb-brightened jet structure. Between 2004 and 2009 the data were recorded at a bit rate of $512 \mathrm{Mbps}$ in left circular polarization (128 MHz bandwidth). The observations in 2015 were performed at a higher bit rate of 2 Gbps and in dual circular polarization, resulting in the total bandwidth of $2 \times 256 \mathrm{MHz}$. The GMVA data from observations before 2015 were reanalyzed for this study. We also included one archival data set from 2014 with VLBA and GBT observations (Hada et al. 2016) in order to enhance the time sampling. The observational details and resulting imaging parameters are summarized in Table 1. 


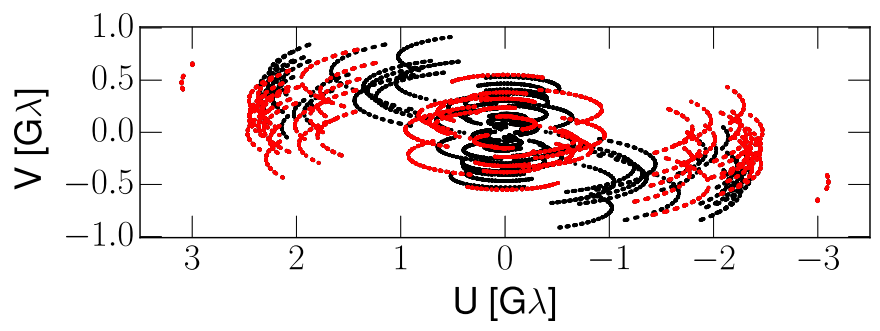

Fig. 1. $(u, v)$-coverage for M 87 from GMVA observations in May 2015. Only scans whose VLBI fringes have been detected are shown. Baselines including high-sensitivity stations (PV, PB, and GB) are marked in red.

The GMVA data were correlated with the Mark IV correlator at the Max-Planck-Institut für Radioastronomie (MPIfR) in Bonn, Germany. The 2015 data were correlated with the DiFX correlator (Deller et al. 2007) at the MPIfR. The post-processing was performed using the Astronomical Image Processing System (AIPS; Greisen 1990) following standard calibration procedures. The fringe fitting was done in two steps. First, we removed constant sub-band delays and phase offsets at different intermediate frequencies (IFs) using high signal-to-noise ratio $(\mathrm{S} / \mathrm{N})$ scans (manual phase-cal). After the phases were aligned across the frequencies, the data were fringe-fitted using the full bandwidth. In this global fitting step, residual single-band and multi-band delays were removed and the fringe rates were determined. For reliable fringe detection we applied a cutoff of $S / N \geq 5$. The a priori amplitude calibration was performed using the station-based system temperature measurements and gainelevation curves. From the elevation dependence of the system temperatures an atmospheric opacity correction was derived and applied for each station. After these basic calibration steps, the data were averaged over frequency and exported to the Difmap VLBI imaging package (Shepherd et al. 1994) where the final coherent time averaging (coherence time $\sim 10 \mathrm{~s}$ ) and data editing was performed. For the imaging, we used the CLEAN algorithm (Högbom 1974) implemented in Difmap. An iterative process of phase and amplitude self-calibration was applied until the rms noise level in the map was minimized. The off-source rms level in each final CLEAN map was estimated using the AIPS task IMEAN, which reads a CLEAN image and fits a Gaussian to the histogram of the pixel values. In addition to the thermal noise, we also estimated the systematic uncertainty of the absolute flux to be $\sim 15 \%$ based on the station gain corrections derived during the amplitude self-calibration.

\section{Results and analysis}

\subsection{Visibilities and resulting images}

In Fig. 1 we show the $(u, v)$-coverage of the GMVA observations in 2015. The inclusion of the large and sensitive telescopes - the IRAM $30 \mathrm{~m}$ telescope at Pico Veleta (PV), the phased Plateau de Bure interferometer (PB), and the GBT - improved the data quality and gave robust fringe detections even on the longest baselines (up to $\sim 3 \mathrm{G} \lambda$ ). In Fig. 2 we show a representative plot of the radial dependence of the visibility amplitudes versus the $(u, v)$-distance. The visibilities drop from $\sim 1 \mathrm{Jy}$ at short $(u, v)$ spacings to $\sim 50-100 \mathrm{mJy}$ at the longest $(u, v)$-distances $(\mathrm{e} . \mathrm{g}$. $3 \mathrm{G} \lambda$, PV, and PB to MK). Figure 3 shows the $86 \mathrm{GHz}$ images of the inner jet region of M 87 between 2004 and 2015 (forward in time from top to bottom). The images show the basic source structure (core-jet morphology with limb-brightening) is similar

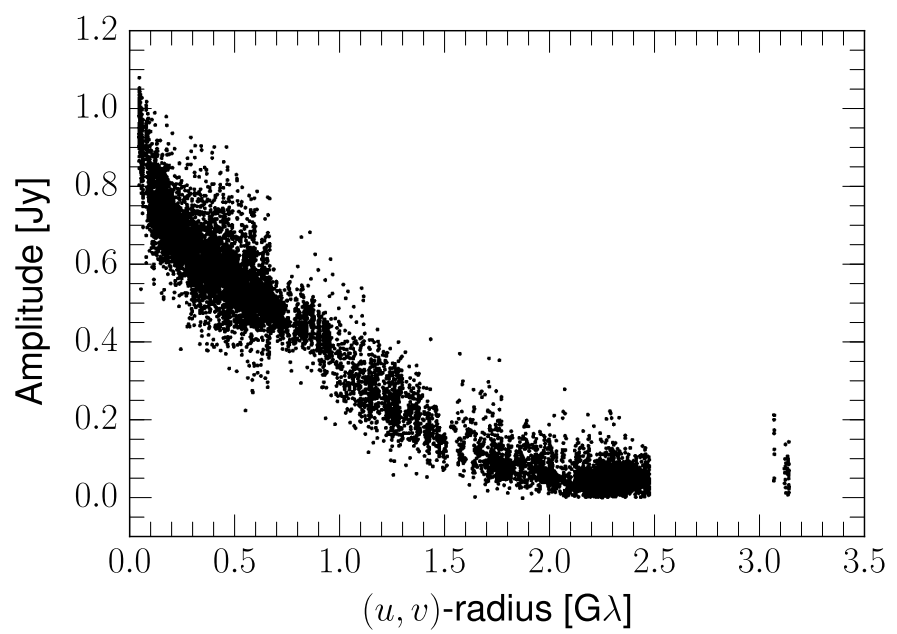

Fig. 2. Radial distribution of the visibility amplitude of M 87 for the GMVA observation at $86 \mathrm{GHz}$ in May 2015. For clarity, the data were binned in $30 \mathrm{~s}$ time intervals.

in all epochs (though the data and image quality differ between observations). In addition to the prominent east-west oriented jet, faint emission east of the bright VLBI core is visible. This feature may be the counter-jet which is also visible at longer wavelengths (Kovalev et al. 2007; Walker et al. 2016). The validity of our counter-jet detection at $86 \mathrm{GHz}$ was tested by applying different phase and amplitude self-calibration schemes which do not allow removal of this feature from the final image. We also point out that the fine-scale structure of the jet and the positions of several bright emission features change with time. We note that our sparse time-sampling does not allow us to robustly cross-identify these bright components across different epochs, which is not unexpected in view of the complex nature of the kinematics in the inner jet (Mertens et al. 2016). We note that for experiment in 2009, no significant signature of flux variability or motion in the jet was detected over a timescale of two days. We therefore combined the two-day experiment into a single visibility data set and made a single image (Fig. 3c).

\subsection{VLBI-core properties}

In order to measure the properties of the VLBI core, we fitted elliptical Gaussian components to the fully calibrated visibilities using the Modelfit task in the Difmap package. The model-fit provided us with the core flux $S_{\text {core }}$, the FWHM size $\psi_{\text {maj,min }}$ along the major and minor axes of the ellipse, and the position angle of the major axis $\mathrm{PA}_{\text {core }}$ for each epoch. To estimate uncertainties in the model-fit parameters, we followed Schinzel et al. (2012), who accounted for the effects of the finite $\mathrm{S} / \mathrm{N}$ and strong side-lobe interference. The ratio of the peak to the rms noise near the core is $\gtrsim 70$ in all epochs. For this $S / N$, the errors of our model-fit parameters are approximately $\sim 15 \%$ and $\sim 20 \%$ for the flux density and the core size, respectively.

The model-fit parameters for the VLBI core are shown in Table 2. In all cases, the FWHM size of the core is larger than $64 \%$ and $23 \%$ of the beam along its minor and major axis, respectively. These sizes are larger than the empirical resolution limit of $1 / 5$ of the beam. If we take the $\mathrm{S} / \mathrm{N}$ of $\sim 70$ into account, model-fit components whose sizes are larger than $\sim 10 \%$ of the beam can be claimed to be spatially resolved (Lobanov 2005; Schinzel et al. 2012). Thus, we conclude that the core of M 87 is spatially resolved (see also Baczko et al. 2016 for a more 


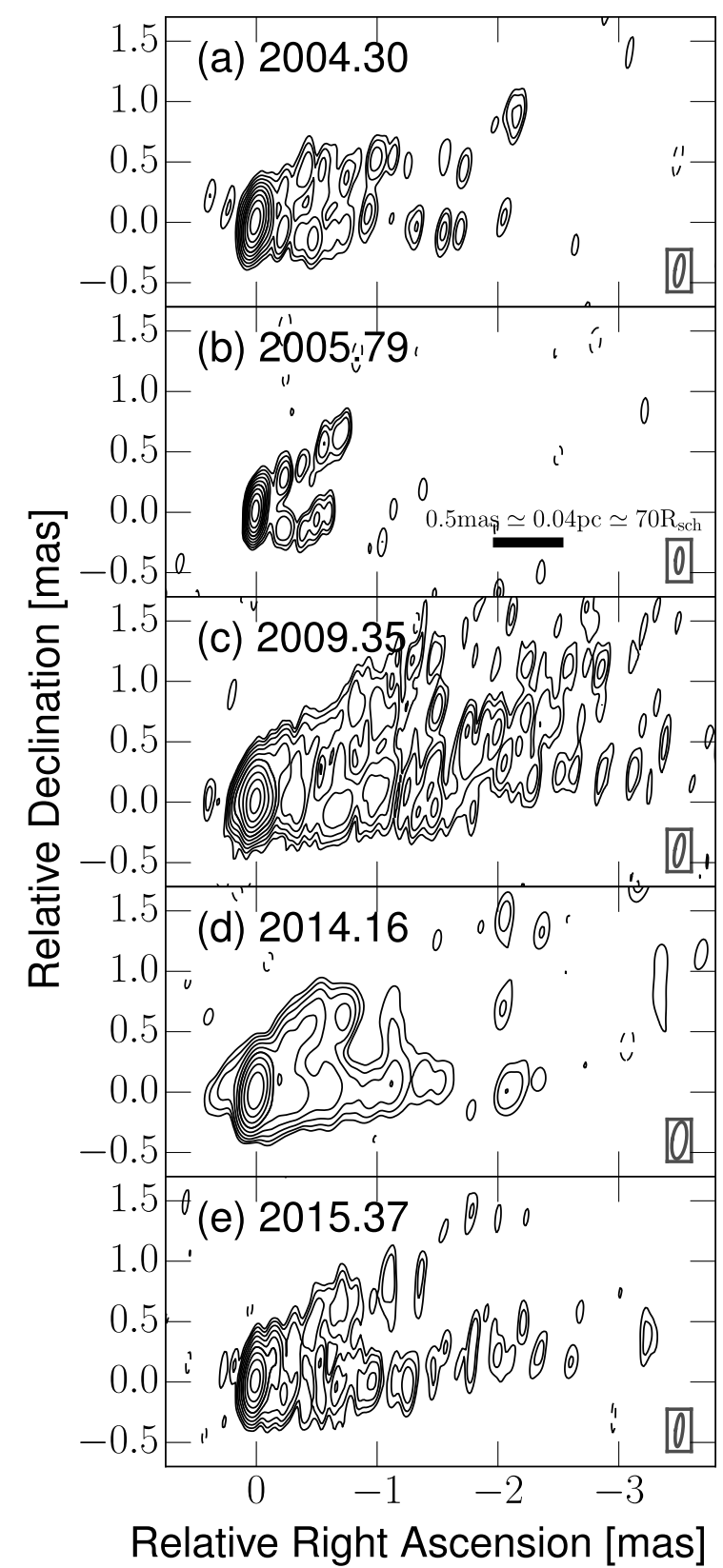

Fig. 3. $86 \mathrm{GHz}$ VLBI images of the inner jet in M 87 obtained from observations between 2004 (top) and 2015 (bottom). The restoring beam is shown as an ellipse in the bottom right corner of each map. The contour levels are $(-1,1,2,4,8, \ldots) \times 1 \mathrm{mJy}^{\text {beam }}{ }^{-1}$.

detailed discussion about determination of the resolution limit of a typical GMVA data set). The flux density of the VLBI core is in the range of $\sim(0.53-0.67) \mathrm{Jy}$ for all observations except those from 2009 when a significantly higher (factor 2) core flux was seen. We checked the amplitude calibration using 3C 273, which was observed in alternate VLBI scans in the same experiment and found no evidence of a systematic amplitude miscalibration. We therefore regard the elevated flux density as intrinsic to the source. Details regarding this particular epoch will be studied in a future publication.

\subsection{Image stacking}

Owing to the sparse time sampling of our observations, we focus on an approach to average all the images in time (image stacking) in order to increase the image sensitivity and study the time-averaged emission, which characterizes the overall shape of the jet launching region. This stacking analysis has also been adopted in other studies, for example in the analysis of the jet structure of $\mathrm{AGN}$ at $15 \mathrm{GHz}$ and other frequencies (e.g., Fromm et al. 2013; MacDonald et al. 2015; Boccardi et al. 2016b; Pushkarev et al. 2017).

For the stacking, the individual jet images (Fig. 3) were restored with a common beam. The restored maps were aligned by the positions of their intensity peaks. The assumption of a stable core position is supported by astrometric VLBA $43 \mathrm{GHz}$ observations of M 87 over multiple epochs (Acciari et al. 2010), which revealed a stationary peak position on scales of $6 R_{\mathrm{sch}}$. We performed the stacking procedure with two different beam sizes: (i) with a larger beam size of $0.1 \times 0.3$ mas in order to recover the faint jet on 1-4 mas core separation, and (ii) with a smaller beam of $0.051 \times 0.123 \mathrm{mas}$, in order to reveal the fine-scale structure on $<1$ mas. The latter beam corresponds to $50 \%$ super-resolution in the N-S direction for uniformly weighted data.

In the top panel of Fig. 4 we show the stacked image with the larger beam (Fig. 4a) and below with the smaller beam (Fig. 4b). The $1 \sigma$ rms level in the higher resolution stacked image is $\sim 0.1 \mathrm{mJy}^{\text {beam }}{ }^{-1}$, making it the deepest and highest resolution view of the M 87 jet structure to date.

\subsection{Transverse emission profile and central emission features}

We measure the transverse width of the jet with cuts perpendicular to the mean jet axis. In Fig. 4c we highlight the transverse intensity profiles in two cuts made at $\sim 0.6$ mas and $\sim 0.8$ mas core separations. The transverse jet profiles appear limb-brightened, with an additional signature of a fainter central emission component that is visible above the $5 \sigma$ image noise level. In order to characterize the brightness of this central component, we calculated the center-to-limb brightness ratio $\rho_{\mathrm{CL}}$. We integrated the fluxes in the central lane and the two limbs over (0.45-0.95) mas core distance and we obtained $\sim 38 \mathrm{mJy}$ and $\sim 15 \mathrm{mJy}$ for each limb and the center, respectively. Accordingly, we find $\rho_{\mathrm{CL}} \sim 0.4 \pm 0.1$. For this calculation we assumed $15 \%$ for the flux density uncertainty for the limb, but $30 \%$ for the fainter central lane. As an illustration, we show the averaged transverse intensity profile in Fig. 5.

\subsection{Ridge line analysis}

In order to analyze the jet base structure using the ridge lines, the following procedure was performed. We took the high-resolution jet image in Fig. $4 \mathrm{~b}$ and rotated the image by $21^{\circ}$ in the clockwise direction assuming that the overall jet position angle $P A_{\mathrm{c}}$ is $-69^{\circ}$ with respect to north. At each core distance $d$, we made slices transverse to the jet and fitted two Gaussians to the two humps in the transverse intensity profile. We were often forced to fit another Gaussian along the jet center axis to improve the fit quality. We began this fitting procedure at a core distance $d \sim 1$ mas and continued fitting the transverse profile down to $d \sim 0.06$ mas. We followed Mertens et al. (2016) in computing the uncertainties in the ridge line analysis. The diameter of the jet $W(d)$ is determined by the distance between the positions of the Gaussian peaks at each $d$. Similarly, we also derive the apparent jet opening angle $\phi_{\text {app }}(d)$ by computing the angle subtended by the two Gaussian peaks. The intrinsic opening angle $\phi_{\text {int }}$ is then given by $\phi_{\text {int }}=2 \arctan \left(\sin \theta \times \tan \left(\phi_{\text {app }} / 2\right)\right)$ (Pushkarev et al. 2017) under the assumption that the extended jet is azimuthally symmetric. 
Table 2. Properties of the VLBI core estimated by elliptical Gaussian model fitting.

\begin{tabular}{cccccccc}
\hline \hline $\begin{array}{c}\text { Epoch } \\
(\text { yyyy/mm/dd })\end{array}$ & $\begin{array}{c}S_{\text {core }} \\
(\mathrm{Jy})\end{array}$ & $\begin{array}{c}\psi_{\text {min }} \\
(\mu \mathrm{as})\end{array}$ & $\begin{array}{c}\psi_{\text {maj }} \\
(\mu \mathrm{as})\end{array}$ & $\begin{array}{c}\sqrt{\psi_{\text {maj }} \times \psi_{\text {min }}} \\
(\mu \mathrm{as})\end{array}$ & $\begin{array}{c}\mathrm{PA}_{\text {core }} \\
\left(R_{\text {sch }}\right)\end{array}$ & $\begin{array}{c}T_{\mathrm{B}} \times \delta \\
(\mathrm{deg}, \mathrm{N} \rightarrow \mathrm{E})\end{array}$ & $\begin{array}{c}\left(\times 10^{10} \mathrm{~K}\right) \\
(8)\end{array}$ \\
\hline $2004 / 04 / 19$ & $0.58 \pm 0.09$ & $60 \pm 12$ & $63 \pm 13$ & $62 \pm 9$ & $8.7 \pm 1.2$ & 29.4 & $2.5 \pm 0.8$ \\
$2005 / 10 / 15$ & $0.53 \pm 0.08$ & $38 \pm 8$ & $88 \pm 18$ & $58 \pm 8$ & $8.1 \pm 1.1$ & -11.0 & $2.6 \pm 0.8$ \\
$2009 / 05 / 09$ & $1.39 \pm 0.20$ & $75 \pm 15$ & $127 \pm 30$ & $102 \pm 15$ & $13.7 \pm 1.9$ & 15.6 & $2.4 \pm 0.8$ \\
$2014 / 02 / 26$ & $0.60 \pm 0.09$ & $75 \pm 15$ & $80 \pm 16$ & $77 \pm 11$ & $10.8 \pm 1.5$ & 18.6 & $1.7 \pm 0.5$ \\
$2015 / 05 / 16$ & $0.67 \pm 0.10$ & $87 \pm 18$ & $91 \pm 18$ & $89 \pm 12$ & $12.5 \pm 1.8$ & 48.0 & $1.4 \pm 0.5$ \\
\hline
\end{tabular}

Notes. The columns show (1) the observing epoch (year/month/day); (2) the core flux (in Jy); (3) and (4) the FWHM core size along the minor and major axis (in $\mu$ as); (5) and (6) the geometrical mean of the core size (in $\mu$ as and $R_{\text {sch }}$, respectively); (7) the position angle of the elliptical core (in deg); and (8) the Doppler-boosted apparent brightness temperature (in $10^{10} \mathrm{~K}$ ).

Figure 6 shows the results of the ridge line analysis. The jet diameter at $d<1$ mas clearly increases with core separation. The overall jet diameter at $d>0.3$ mas is $W \sim 0.3-0.7$ mas. At smaller core separations ( $d<0.2$ mas, $90 R_{\text {sch }}$ projected), the collimation profile slightly flattens and the jet diameter does not sharply decrease. At the jet base ( $d=0.06$ mas), the jet width is $0.29 \pm 0.09$ mas $\left(40.8 \pm 12.3 R_{\text {sch }}\right)$ with an apparent opening angle of $\phi_{\text {app }}=127^{\circ} \pm 22^{\circ}$. Depending on the jet viewing angle, the intrinsic jet opening angle is $\phi_{\text {int }}=63.6^{\circ} \pm 25.0^{\circ}\left(90^{\circ} \pm 28^{\circ}\right)$ for a jet viewing angle $\theta=18^{\circ}\left(30^{\circ}\right)$.

The asymptotic structure in the measured jet width $W$ versus the distance from the central engine $z$ was fit with a powerlaw model $W(z) \propto z^{k}$, where $k$ is a dimensionless index which parameterizes the jet expansion and acceleration within theoretical models (e.g., Komissarov et al. 2007; Lyubarsky 2009). It is important to note that the core separation $d$ is not necessarily the same as the distance from the central engine $z$ because of the jet opacity (e.g., Lobanov 1998). Hence, we associate the core separation $d$ with the distance from the central engine by $z=\epsilon+d$, where $\epsilon$ is the unknown offset between the BH and the $86 \mathrm{GHz}$ core (see Fig. 7). We adopted $\epsilon \leq 41 \mu$ as based on the results of Hada et al. (2011), where the authors performed astrometric VLBA observations toward M 87 at $2.3-43.2 \mathrm{GHz}$ and estimated the distance between the intensity peak and the jet apex at $43 \mathrm{GHz}$. Then we obtained $W$ as function of $z$ and the power-law model was fit to $W(z)$.

We find a jet expansion rate of $k=0.469 \pm 0.019$ when we ignore the core shift at $86 \mathrm{GHz}$ (i.e., $\epsilon=0$ ). With a nonzero core-shift correction $(\epsilon \neq 0)$, we find $k \sim 0.47-0.51$ with a mean value of $k=0.498 \pm 0.025$ (the error represents uncertainties in both the core position and the statistical fitting). Both fits have a reduced chi square of $\chi_{\text {red }}^{2} \sim 0.51$. This is in agreement with previous values of $k=0.56-0.60$ (Asada \& Nakamura 2012; Hada et al. 2013; Mertens et al. 2016) within $3 \sigma$ uncertainty levels. To demonstrate the goodness of fit, we calculated the fractional difference between the model and the data, $\Delta W / W=\left(W_{\text {obs }} / W_{\text {fit }}-1\right)$, where $W_{\text {obs }}$ and $W_{\text {fit }}$ are the observed and model jet widths, respectively. The results are shown in the bottom panel of Fig. 6. It can be seen that the fractional difference is nearly zero, but starts to grow within $d \leq 0.2$ mas.

\subsection{Ratio of jet to counter-jet}

We measured the integrated jet and counter-jet flux density at core separations of $0.2-0.5$ mas (projected distances of $\sim 28-140 R_{\text {sch }}$ ) using the jet image in Fig. 4 a. For the main jet, we placed a large box covering all three elements of the approaching jet (i.e., the northern limb, the southern limb, and the central emission). Another box of the same size was placed in the counter-jet region. The measured flux density of the jet and the counter-jet were $\sim 95 \mathrm{mJy}$ and $\sim 3.5 \mathrm{mJy}$, respectively. Accordingly, we obtained the jet-to-counter-jet ratio $R=27.1 \pm 9.1$ (assuming $15 \%$ and $30 \%$ of flux uncertainties for the approaching and the receding jets, respectively). We note the observational evidence for limbbrightening in the counter-jet at $43 \mathrm{GHz}$ (Walker et al. 2016). This is not seen in our data, possibly due to sensitivity limitations and the higher frequency. Therefore, our counter-jet flux could be underestimated. In order to correct for this, we measured the integrated flux of only the southern limb of the approaching jet at the same core distances assuming that we see only the northern edge of the limb-brightened counter-jet. The integrated flux of the southern limb of the approaching jet was reduced to $\sim 60 \mathrm{mJy}$. This lowers the jet-to-counter-jet ratio to $R=17 \pm 6$.

We also measured the jet-to-counter-jet ratio variation in the longitudinal direction using individual pixel values. For this, we cut the jet longitudinally through the counter-jet, the core, and the southern limb of the approaching jet. We took the jet image in Fig. 4a to obtain a smoother jet intensity gradient. For a more reliable measurement, we used only pixel values over the $7 \sigma$

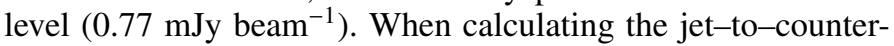
jet ratio as a function of the distance from the central engine, using the same jet apex to core offset $\epsilon$ may affect our measurements (see Fig. 7 for illustration). Therefore, we adopted the same $\epsilon \leq 41 \mu$ as in a direction east of the VLBI core (highlighted in gray in Fig. 7; see Sect. 3.5 for details) and calculated the brightness ratio at the corresponding jet distance. We accounted for thermal noise, systematic amplitude error, and the dispersion in the $R$ profile at each distance $z$ due to the uncertainty in the central engine position. We further excluded $R$ values measured within 0.1 mas of the central engine because of the relatively large beam size in the image presented in Fig. 4a. The result is shown in Fig. 8. The $R$ measured within $\sim 0.2$ mas from the central engine is largely affected by the positional uncertainty of the $86 \mathrm{GHz}$ VLBI core, $\epsilon$, causing $R$ to vary by a factor of $\sim 5$. Nevertheless, we find that a single constant value of the jet-tocounter-jet ratio is not suitable in describing the relatively large variation in $R(R=1-10$ near 0.1 mas and $R=10-25$ at larger distances).

\section{Discussion}

\subsection{Physical conditions in the VLBI core region}

The VLBI core brightness temperature parameterizes the physical conditions within compact energetic jet components 

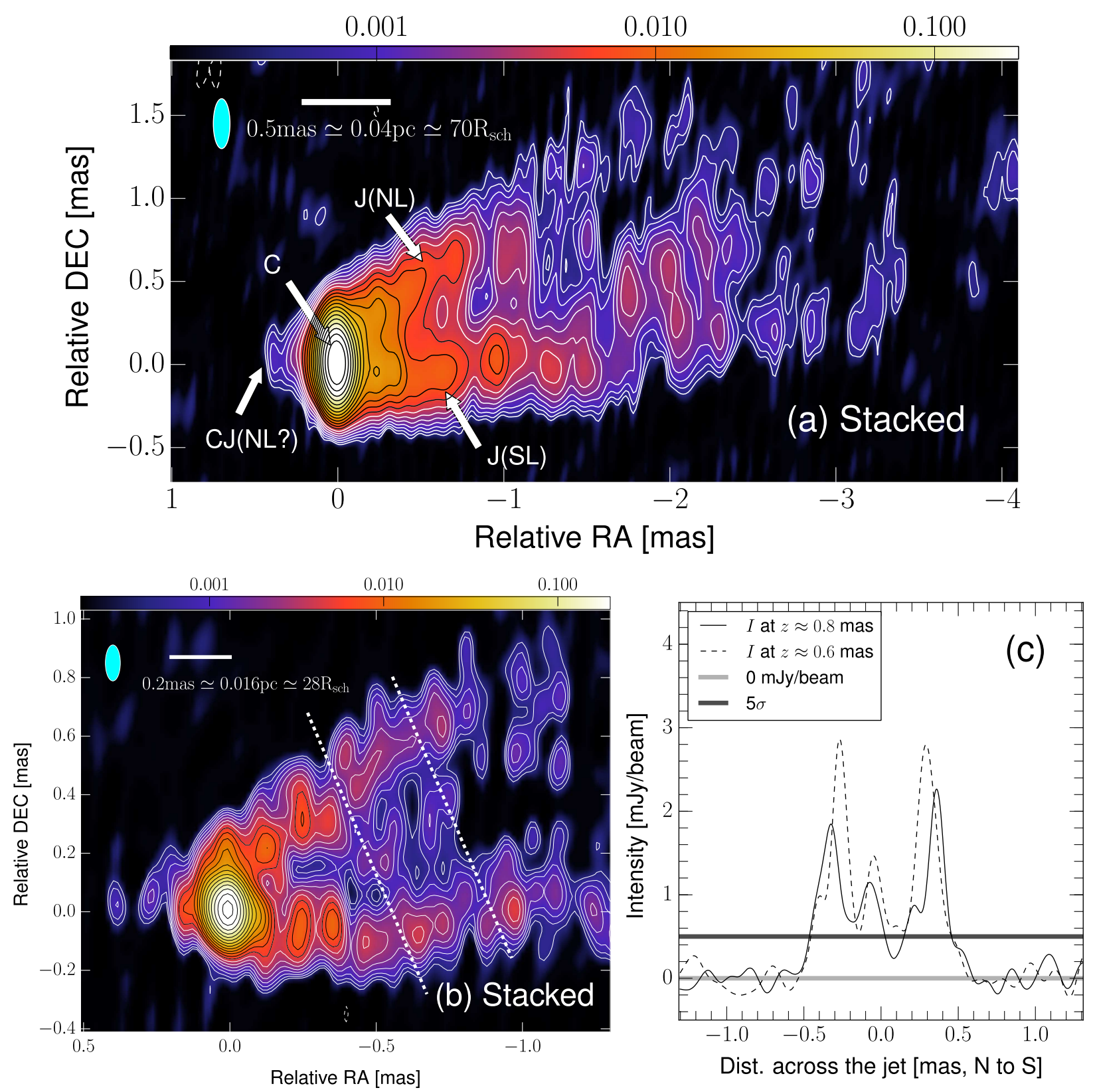

Fig. 4. Stacked M 87 jet images and transverse intensity profiles. Panel a: image with a restoring beam of $0.3 \times 0.1$ mas. The core $(C)$, northern/southern limbs (NL/SL) of the jet (J), and the counter-jet (CJ) are indicated by white arrows. Panel $b$ : as in panel a, but restored with a smaller beam of $0.123 \times 0.051$ mas and zoomed in on the inner region. The colorbars indicate total intensities in units of Jy beam ${ }^{-1}$. Contour levels are $(-1,1,1.4,2,2.8, \ldots) \times 0.47 \mathrm{mJy}_{\text {beam }}{ }^{-1}$. The white bars indicate projected linear distance scales for M 87 . The white dashed lines denote the position of the slices in panel $c$. The restoring beams are indicated by the cyan ellipses in the top left corner of each panel. Panel $c$ : transverse jet intensity profiles measured using the higher resolution image in Fig. 4b (starting from north to south). The dark solid/broken lines are the measured intensity at $\sim 0.8 / 0.6$ mas core distance, respectively. The light gray line is the zero intensity level and the dark thick gray line indicates the $5 \sigma$ level.

(e.g., Kovalev et al. 2005; Lee et al. 2016). We calculate the intrinsic brightness temperature of the core in the source rest frame, $T_{\mathrm{B}}$, by

$T_{\mathrm{B}}=1.22 \times 10^{12} \frac{S_{\text {core }}}{v^{2} \psi_{\text {maj }} \psi_{\text {min }}} \frac{(1+z)}{\delta} \quad \mathrm{K}$

(Lee et al. 2016), where $v$ is the observing frequency in $\mathrm{GHz}$, $S_{\text {core }}$ is in Jy, $\psi_{\text {maj }}$ and $\psi_{\text {min }}$ are in mas, $z=0.00436$ is the redshift of M 87 (Smith et al. 2000), and $\delta$ is the Doppler factor. The observed apparent brightness temperature, $T_{\mathrm{B} \text {,app }}=T_{\mathrm{B}} \times \delta$, is shown in Table 2 . We find that the $T_{\mathrm{B} \text {,app }}$ is generally quite low.
Remarkably, the $T_{\mathrm{B}, \mathrm{app}}$ is always lower than $T_{\mathrm{eq}} \sim\left(5 \times 10^{10}\right) \mathrm{K}$, which is often referred to as the brightness temperature of a plasma in which the energy density of the magnetic field $u_{\mathrm{B}}$ is equal to that of the particles $u_{\mathrm{p}}$ (i.e., the "equipartition brightness temperature"; Readhead 1994). The intrinsic brightness temperature will be even lower if Doppler boosting is accounted for.

In order to examine whether the equipartition brightness temperature for the non-thermal electrons in the jet of M 87 is higher or lower than $5 \times 10^{10} \mathrm{~K}$, we explicitly calculate the equipartition brightness temperature in the following manner, using the analysis presented by Singal (2009). Assuming equal energy density for particles $u_{\mathrm{p}}$ and magnetic field $u_{\mathrm{B}}$ in the jet 


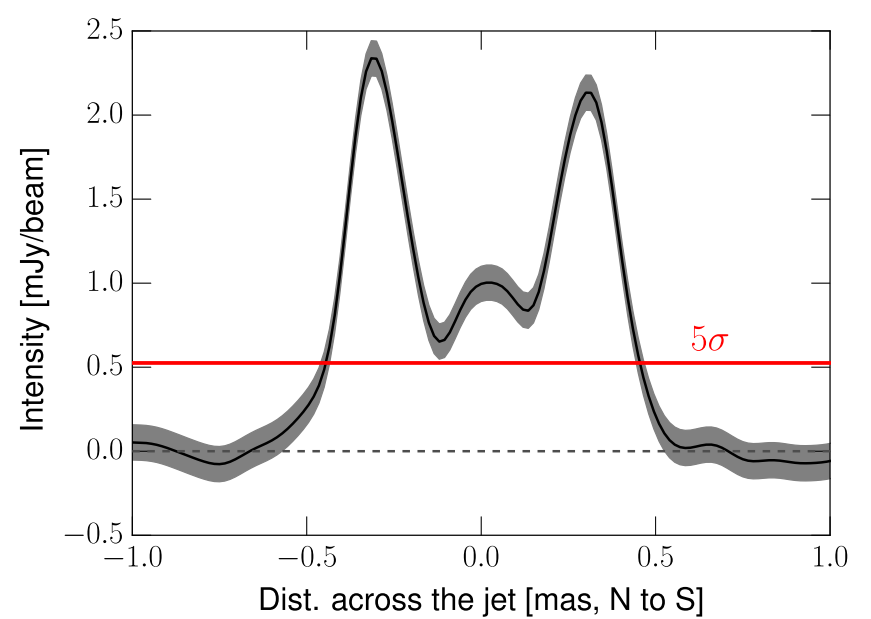

Fig. 5. Transverse jet intensity profile obtained by averaging the jet emission over $\sim 0.5-0.9$ mas core distance. The dark solid line is the measured intensity, the shaded region indicates $1 \sigma$ image rms noise level, and the red line denotes the corresponding $5 \sigma$ level.

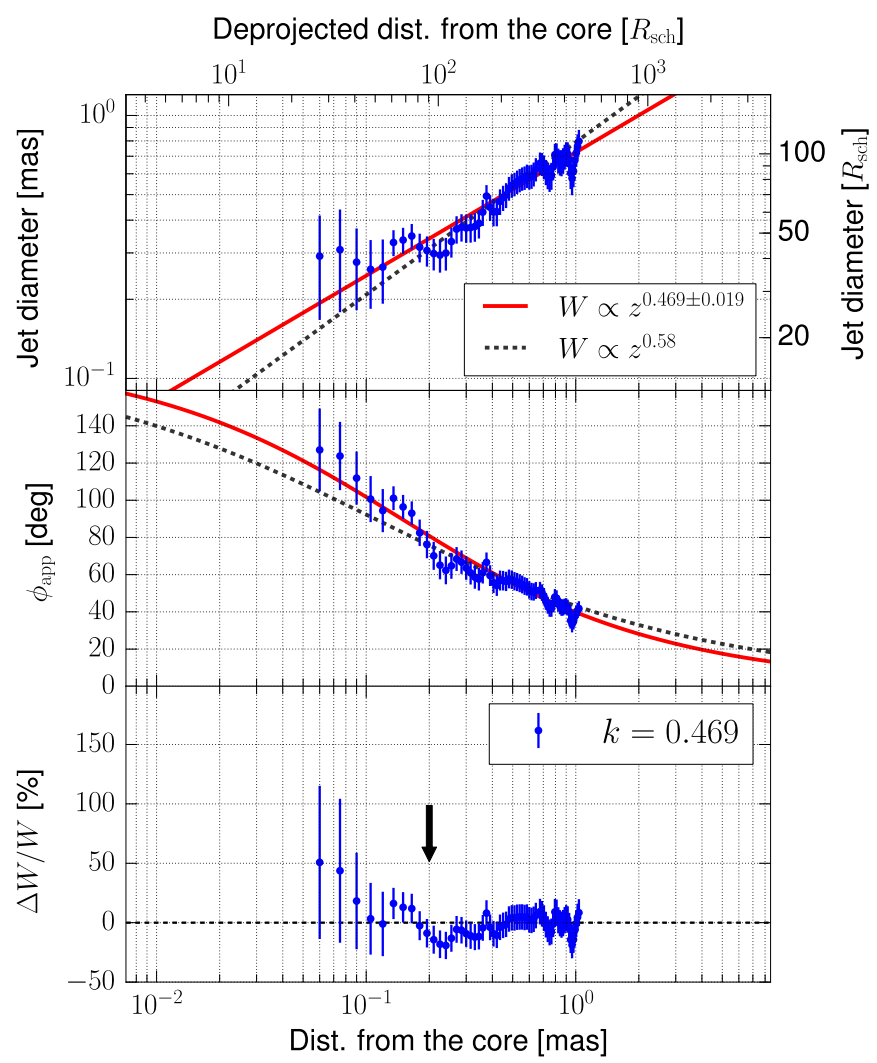

Fig. 6. M 87 jet base collimation profile plotted vs. the core separation $d$. Top: jet width and power-law fit. The blue data points are the measurements, the red solid line is the best power-law fit to the data, and the gray broken line is a fit with a fixed slope of $k=0.58$. Middle: same as above, but for the apparent opening angle. Bottom: fractional difference between the observed diameter and the power-law model with a corresponding slope, $k \approx 0.47$. The arrow indicates a core distance where the fractional difference starts to grow significantly.

frame (i.e., without Doppler boosting), we can rewrite Eq. (3) of Singal (2009) to express $T_{\mathrm{B} \text {,eq }}$ in the source frame as

$T_{\mathrm{B}, \mathrm{eq}}=t(\alpha) 10^{11}\left[\left(\frac{s}{\mathrm{pc}}\right)\left(\frac{\nu_{\text {turn }}}{\mathrm{GHz}}\right)^{1.5+\alpha}\right]^{1 / 8} \mathrm{~K}$,

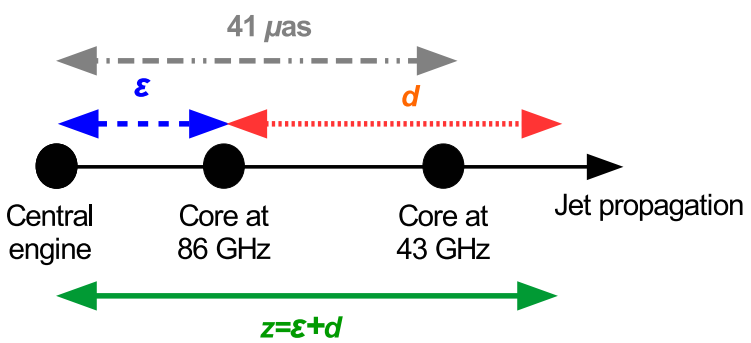

Fig. 7. Illustration of the central engine and the $86 \mathrm{GHz}$ core geometry described in Sect. 3.5.

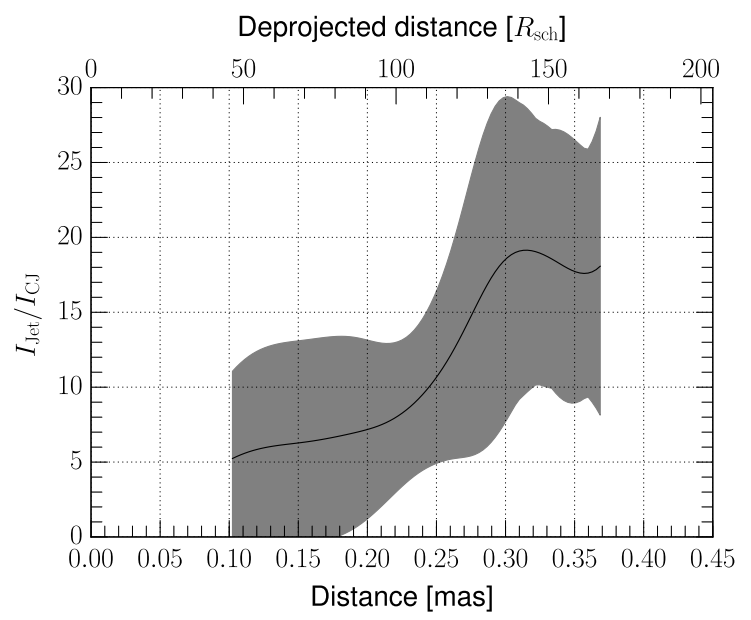

Fig. 8. Jet-to-counter-jet intensity ratio $(R)$ measured as described in the text. The solid line shows the measured values and the shaded region indicates the uncertainties. A viewing angle of $\theta=18^{\circ}$ was used to calculate the deprojected distances.

where $\alpha$ is the spectral index $\left(S \propto v^{+\alpha}\right.$; we note that the sign convention is different from Singal 2009), $t(\alpha)$ is a numerical function of the spectral index tabulated in Singal (2009) $(t(\alpha) \sim 0.3-0.7$ for $\alpha \sim-0.3$ to -1.5$), s$ is the linear size of the emitting region, and $v_{\text {turn }}$ is the synchrotron turn-over frequency. In the observer's rest frame, $T_{\mathrm{B}, \mathrm{eq}}$ is boosted by the Doppler factor $\delta$ (Readhead 1994). For $s$ we adopt the mean FWHM size of the VLBI core ${ }^{2}, 77 \mu$ as $\left(\sim 11 R_{\text {sch }} \sim 0.006 \mathrm{pc}\right)$. The exact values of $v_{\text {turn }}$ and $\alpha$ on a scale $\lesssim 11 R_{\text {sch }}$ are not well determined due to the lack of simultaneous multi-frequency VLBI observations at high resolution. Nevertheless, we compare the time-averaged core flux at $86 \mathrm{GHz}(\sim 0.5 \mathrm{Jy})$ and at $230 \mathrm{GHz}$ ( 1 Jy; Doeleman et al. 2012; Akiyama et al. 2015) and find an indication of an inverted spectrum between $86 \mathrm{GHz}$ and $230 \mathrm{GHz}$. Also, Broderick \& Loeb (2009) suggested a turnover frequency $v_{\text {turn }} \sim 230 \mathrm{GHz}$ and a spectral index $\alpha \sim-1.0$ using other available total flux density measurements. Hence, we adopt $v_{\text {turn }}=230 \mathrm{GHz}$ and $\alpha=-1.0$ in our analysis. In addition, we note that Eq. (2) is insensitive to the exact value of $v_{\text {turn }}$ and $\alpha$ due to the $1 / 8$ power dependence. The Doppler factor is given by $\delta=1 / \Gamma(1-\beta \cos \theta)$ where $\Gamma$ is the bulk Lorentz factor, $\beta$ is the intrinsic jet speed, and $\theta$ is the jet viewing angle. We adopt the apparent jet speed of $\sim 0.5 \mathrm{c}$ and the viewing angle of $\theta=18^{\circ}$ (Hada et al. 2016; Mertens et al. 2016), which gives $\delta \sim 2$. Computing Eq. (2), we obtain a value of $T_{\mathrm{B} \text {,eq }}$ in the observer's frame of $\approx 7.7 \times 10^{10} \mathrm{~K}$. For consistency, we also calculate $T_{\mathrm{B} \text {,eq }}$ using

\footnotetext{
2 Marscher (1983) suggested a correction factor of 1.8 for the Gaussian to spherical size conversion. This correction changes the $T_{\mathrm{B}, \mathrm{eq}}$ by a factor of only $1.8^{1 / 8} \sim 1.08$. Thus, we ignore this size correction.
} 
Table 3. Brightness temperature ratio $T_{\mathrm{B}, \mathrm{eq}} / T_{\mathrm{B}}$ with the equipartition brightness temperature of $2 \times 10^{11} \mathrm{~K}$ in the observer's rest frame and the magnetic field strength in units of $B_{\text {eq }}$.

\begin{tabular}{ccc}
\hline \hline $\begin{array}{c}\text { Epoch } \\
(1)\end{array}$ & $\begin{array}{c}T_{\mathrm{B}, \mathrm{eq}} / T_{\mathrm{B}} \\
(2)\end{array}$ & $\begin{array}{c}B / B_{\mathrm{eq}}(\times 10) \\
(3)\end{array}$ \\
\hline $2004 / 04 / 19$ & $8 \pm 3$ & $6.4 \pm 4.1$ \\
$2005 / 10 / 15$ & $8 \pm 2$ & $6.1 \pm 3.9$ \\
$2009 / 05 / 09$ & $8 \pm 3$ & $6.9 \pm 4.4$ \\
$2014 / 02 / 26$ & $12 \pm 4$ & $15 \pm 9$ \\
$2015 / 05 / 16$ & $14 \pm 5$ & $21 \pm 13$ \\
\hline
\end{tabular}

Notes. The columns show (1) the observing epoch, (2) the $T_{\mathrm{B}}$ ratio, and (3) the magnetic field strength ratio.

Eq. (4b) in Readhead (1994), which yields $T_{\mathrm{B}, \mathrm{eq}} \approx 3.5 \times 10^{11} \mathrm{~K}$ in the same frame.

Accordingly, the equipartition brightness temperature is larger than the observed values by nearly an order of magnitude. It is worth noting that the broadband spectral energy decomposition of the core of M 87 shows a large dominance of the jet emission. In particular, emission from non-thermal electrons in the jet dominate over the emission from thermal (and non-thermal) electrons in the surrounding accretion disk (Broderick \& Loeb 2009; Prieto et al. 2016). We also recall that the observed brightness temperature is still higher than the effective temperature of an electron $m_{\mathrm{e}} c^{2} / k_{\mathrm{B}} \sim 6 \times 10^{9} \mathrm{~K}$. Therefore, the core brightness temperature is a good representation of the microscopic energy of the non-thermal electrons in the jet. Hence, we conclude that in the VLBI core at $86 \mathrm{GHz}$ and on spatial scales of $\sim 11 R_{\mathrm{sch}}$, the magnetic field energy density in the jet is higher than that of the non-thermal particles.

The corresponding magnetic field strength $B$ in the jet can be estimated by $B=B_{\text {eq }}\left(T_{\mathrm{B}, \mathrm{eq}} / T_{\mathrm{B}}\right)^{2}$ (Readhead 1994$)$, where $B_{\mathrm{eq}}$ is the equipartition magnetic field strength. In Table 3 we list $T_{\mathrm{B} \text { eq }} / T_{\mathrm{B}}$ and $B / B_{\text {eq }}$, adopting an equipartition brightness temperature of $T_{\mathrm{B} \text {,eq }}=2 \times 10^{11} \mathrm{~K}$. If the characteristic equipartition magnetic field strength in the VLBI core region of M 87 matches the estimates for other AGN on larger scales (on the order of $\sim 1 \mathrm{G}$, Pushkarev et al. 2012), the true magnetic field strength can lie in the range of $61-210 \mathrm{G}$ at the $86 \mathrm{GHz}$ jet base and even higher when approaching closer to the central engine. This agrees with an independent estimate from Kino et al. (2015), who also obtained $B \sim 100 \mathrm{G}$. We note that such a strong magnetic field seems to be present in some other AGN-jet systems (Zamaninasab et al. 2014; Martí-Vidal et al. 2015; Baczko et al. 2016). Following Readhead (1994), the energy density ratio $u_{\mathrm{p}} / u_{\mathrm{B}}$ is obtained by

$\frac{u_{\mathrm{p}}}{u_{\mathrm{B}}}=\left(\frac{T_{\mathrm{B}, \mathrm{eq}}}{T_{\mathrm{B}}}\right)^{-17 / 2}$.

For the equipartition brightness temperature $T_{\mathrm{B} \text {; eq }} \sim 8 \times 10^{10} \mathrm{~K}$, we find $-6 \lesssim \log \left(u_{\mathrm{p}} / u_{\mathrm{B}}\right) \lesssim-4$. This ratio is even lower if the equipartition brightness temperature is higher. Thus, the inner jet of M 87 appears to be magnetically dominated.

We note that the above calculations assume a negligible energy contribution from thermal particles in the accretion flow, which may be entrained in the sheath of the jet (e.g., Mościbrodzka et al. 2016). The brightness temperature of M 87 is also quite low compared to typical values found in other sources at this frequency $\left(T_{\mathrm{B}, \mathrm{app}} \sim 10^{11} \mathrm{~K}\right.$; Lee et al. 2016; Nair et al. 2018), perhaps indicating that the non-thermal particles should be at least mildly relativistic. In this situation, the non-thermal particles in the low-energy tail of the particle density distribution cannot be easily distinguished from those in the thermal particle density distribution. For instance, we recall that near the $86 \mathrm{GHz}$ VLBI core the jet plasma is still opaque to synchrotron radiation (see also Kim et al. 2018). The selfabsorbed synchrotron radiation may heat the low-energy particles, which will in turn modify the spectral shape of the particle energy distribution at lower energies (Ghisellini et al. 1988).

We can make an estimate for the impact of the thermal particles based on a couple of assumptions. First, the number density of thermal particles, $n_{\mathrm{th}}$, in the hot accretion flow of M 87 would be two orders of magnitude larger than that of non-thermal particles, $n_{\text {nth }}$, in order to reproduce the observed source spectrum (e.g., Broderick \& Loeb 2009). The ratio of number densities would remain the same in the jet if all the particles in the jet were only supplied by the accretion flow. We note that various particle acceleration mechanisms may operate close to the jet base and could make the number density of non-thermal particles higher than we assume here (e.g., McKinney 2006; $\mathrm{Pu}$ et al. 2017). Second, the temperature, $T_{\mathrm{i}}$, of energetic ions in the hot accretion flow is nearly virial and can be described by $T_{\mathrm{i}} \sim 10^{12} \times\left(z / R_{\mathrm{sch}}\right)^{-1} \mathrm{~K}$, where $z$ is the distance from the central BH (Yuan \& Narayan 2014). The electron temperature in such a model is generally much lower than the ions, and thus electrons would not significantly contribute to the internal energy of the gas. If a part of the accreting matter is entrained within the jet at the location of the $86 \mathrm{GHz}$ core and accounts for the internal energy of the thermal particles in the jet, the energy of the thermal particles in the jet, $u_{\mathrm{th}}$, is approximately $u_{\mathrm{th}} \sim n_{\mathrm{th}} k_{\mathrm{B}} T_{\mathrm{i}}$, where $k_{\mathrm{B}}$ is the Boltzmann constant. The $86 \mathrm{GHz}$ core is presumably located at $z \sim 10 R_{\text {sch }}$ from the BH (e.g., Hada et al. 2011, 2016) and the corresponding temperature is be $T_{\mathrm{i}} \sim 10^{11} \mathrm{~K}$. For the non-thermal particles in the jet, the energy density $u_{\text {nth }}$ can be estimated by $u_{\mathrm{nth}} \sim n_{\mathrm{nth}} \gamma m_{\mathrm{e}} c^{2} \sim 6 \times 10^{9} \gamma n_{\mathrm{nth}} k_{\mathrm{B}}$, where $\gamma$ is a characteristic particle Lorentz factor. For mildly relativistic nonthermal particles, we can presume $\gamma$ is on the order of unity. Therefore, the energy density ratio between the thermal and nonthermal particles is $u_{\mathrm{th}} / u_{\mathrm{nth}} \approx\left(10^{11} / 6 \times 10^{9}\right)\left(n_{\mathrm{th}} / n_{\mathrm{nth}}\right) \sim 10^{3}$. According to our assumption, the jet particle energy density may be dominated by thermal particles. However, this still does not change the larger dominance of the magnetic field energy in the total energy budget $\left(-3 \lesssim \log \left(u_{\mathrm{p}} / u_{\mathrm{B}}\right) \lesssim-1\right.$ with $\left.u_{\mathrm{p}} \rightarrow 10^{3} u_{\mathrm{p}}\right)$. This implies that the magnetic energy dominates the jet on this spatial scale.

Another important implication of the observed low brightness temperature at $86 \mathrm{GHz}$ is that it is lower than the inverseCompton limit $T_{\mathrm{B}}\left(\sim 10^{12} \mathrm{~K}\right.$; Kellermann \& Pauliny-Toth 1969), which limits the production of high-energy photons. Thus, the inverse-Compton scenario disfavors the compact $86 \mathrm{GHz}$ VLBI core region $\left(\sim 11 R_{\mathrm{sch}}\right)$ as the dominant source of gamma-ray or $\mathrm{TeV}$ photons. Interestingly, the timing analysis of $\mathrm{TeV}$ and $\gamma$-ray events associated with the M87 nuclear region constrain the high-energy photon production site to be as large as $\sim 20-50 R_{\text {sch }}$ (Acciari et al. 2010; Abramowski et al. 2012; Hada et al. 2014), which is 2-5 times larger than the size of the $86 \mathrm{GHz}$ VLBI core.

\subsection{Estimating the size of the jet launching region}

With high-resolution $86 \mathrm{GHz}$ VLBI jet images, it is possible to estimate the diameter of the jet base $D_{0}$ by using the width and collimation profile of the outflow. For this we assume a selfsimilar jet and that the observed power-law dependence can be 


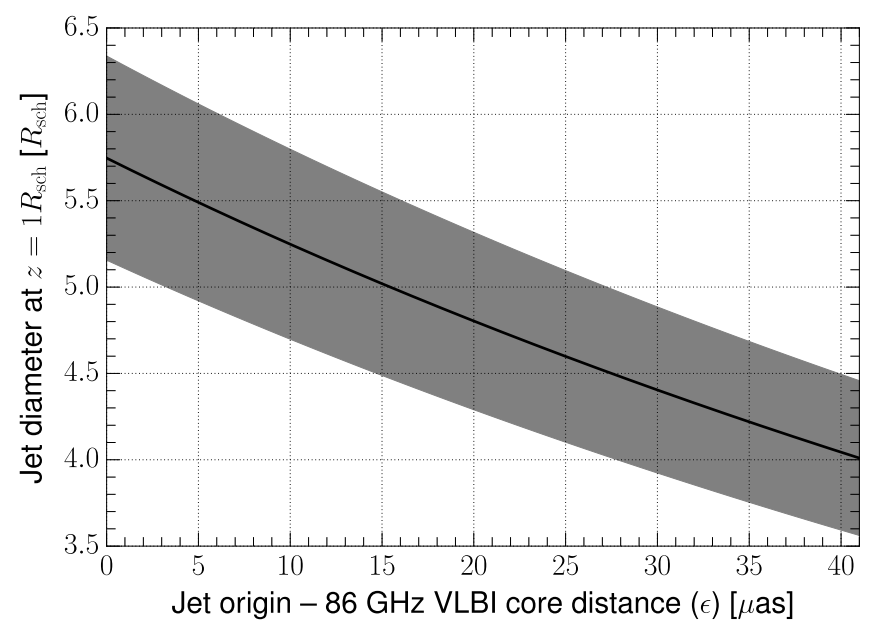

Fig. 9. Diameter of the jet base at a distance of $1 R_{\text {sch }}$ from the BH center as a function of positional offset $\epsilon$ between $\mathrm{BH}$ and the $86 \mathrm{GHz}$ VLBI core (larger separation for larger $\epsilon$ ). The solid line marks the diameter (width) of the jet base and the shaded area denotes the $1 \sigma$ uncertainty from the fitting.

used to back-extrapolate the jet width to its origin near the event horizon. We then determine the collimation profile to $z=1 R_{\mathrm{sch}}$, keeping the unknown separation $\epsilon$ between the BH and the VLBI core at $86 \mathrm{GHz}$ as a free parameter $(\epsilon \leq 41 \mu \mathrm{as}$; see Sect. 3.5 and Fig. 7).

In Fig. 9 we show the estimated size of the jet base versus the displacement $\epsilon$ of the VLBI core from the BH. The width of the jet base is in the range of $\sim(4.0-5.5) R_{\mathrm{sch}}$, with a statistical uncertainty of $\sim 0.5 R_{\mathrm{sch}}$. This small size is consistent with the upper limit given by the $86 \mathrm{GHz}$ VLBI core FWHM size, which is $\sim 11 R_{\text {sch }}$. It is also consistent with a circular Gaussian model fit size estimate of $R_{0}=(5.6 \pm 0.4) R_{\text {sch }}$ (or equivalently $40 \pm 1.8 \mu$ as) obtained at $230 \mathrm{GHz}$ with EHT observations (Doeleman et al. 2012), although the $230 \mathrm{GHz}$ size measurements can be model-dependent due to the limited $(u, v)$ coverage in the early EHT experiments.

Physically, this jet base size is comparable to the diameter of the innermost stable circular orbit (ISCO) for a non-spinning $\mathrm{BH} D_{\text {ISCO }} \sim 6 R_{\text {sch }}$ and it is much larger than $D_{\text {ISCO }}$ for a maximally spinning $\mathrm{BH}$ with prograde disk rotation $\left(D_{\mathrm{ISCO}}=1 R_{\mathrm{sch}}\right)$. On the other hand, the diameter of the ergosphere in a rotating $\mathrm{BH}$ ranges between $(1-2) R_{\text {sch }}$ (Komissarov 2012). This suggests that the diameter of the jet base of M 87 matches the dimensions of the innermost portion of the accretion disk. It is interesting to note that for some other nearby AGN-jets (e.g., 3C 84, Giovannini et al. 2018; Cygnus A, Boccardi et al. 2016a) the jet base appears to be much wider (100 s of $\left.R_{\text {sch }}\right)$.

We also highlight a somewhat higher value of $D_{0} \simeq(9.6 \pm 1.6) R_{\text {sch }}$, which Mertens et al. (2016) determined for M 87 at $43 \mathrm{GHz}$ via an independent analysis. The difference in values for the jet base at 86 and $43 \mathrm{GHz}$ might be simply due to systematics, but several other physical explanations are also possible. One possibility is that multi-frequency VLBI observations see radio emission coming from different layers of a transversely stratified jet, i.e., with stronger emission from the outer layers at lower frequencies. For instance, we refer to VLBI observations of Cygnus $A$ at $5 \mathrm{GHz}$ and $86 \mathrm{GHz}$, which show significantly different jet transverse widths (Carilli et al. 1991; Boccardi et al. 2016a).

An alternate interpretation of these differing values at 86 and $43 \mathrm{GHz}$ could also be attributed to the jet viewing angle.
The jet of $\mathrm{M} 87$ has a small viewing angle of $15^{\circ}-30^{\circ}$. A transverse velocity stratification of the layered jet sheath would lead to differential Doppler boosting (see Komissarov 1990). This effect depends on the viewing angle and could lead to different jet widths at different frequencies. The accretion disk geometry is another factor. The accretion flow in the center of M 87 is believed to be geometrically thick and the scale height of the jet launching point could also be model-dependent (see Yuan \& Narayan 2014 for a review).

It is important to note that the geometrically wide origin of the sheath does not exclude the existence of a more narrow relativistic spine which may be rooted in the $\mathrm{BH}$ ergosphere (Blandford \& Znajek 1977). An apparently faint, Doppler-deboosted ultra-relativistic spine located at the center of the jet could explain the observed limb-brightening (e.g., Komissarov 1990). The high-energy emission observed from M 87 also favors the presence of a fast spine surrounded by the slower sheath (see discussions in Abramowski et al. 2012). For instance, more recent measurements by the EHT with longer baselines (Krichbaum et al. 2014) and theoretical modeling of the jet base region (Kino et al. 2015) provide hints of the existence of a compact emission region significantly smaller than originally inferred from EHT observations with less extended baseline coverage (Doeleman et al. 2012; Akiyama et al. 2015). We also point to complex transverse jet intensity profiles shown by recent deep imaging of M 87, which may be consistent with the idea of the multiple jet layers with different origins (Asada et al. 2016; Hada 2017).

\subsection{Implication of the large intrinsic opening angle in the innermost region}

In the jet acceleration and collimation zone, the relationship between the jet opening angle and the jet speed is important. In particular, Hada et al. (2016) suggested that several reconfinement nodes may form or a jet breakout from a dense atmosphere might occur near the base of the jet in M 87. If this is the case, the expansion and acceleration of the jet will be significantly different from the collimation acceleration scenario (Komissarov et al. 2007; Lyubarsky 2009). For instance, for a sufficiently narrow jet of a given speed the jet-crossing time can be short enough for pressure disturbances from the ambient medium to propagate across the jet (i.e., causally connected). On the other hand, an opening angle that is too wide makes the jet less sensitive to the ambient pressure. The latter case could lead to a different jet acceleration mechanism such as the rarefaction acceleration (e.g., Tchekhovskoy et al. 2010; Komissarov et al. 2010).

We examine the relationship between the intrinsic opening angle $\phi_{\text {int }}$ and the bulk Lorentz factor $\Gamma$ at the core separation $d=60 \mu$ as $\left(27 R_{\text {sch }}\right)$. According to Komissarov et al. (2009), a causally connected jet should satisfy

$\Gamma \phi_{\text {int }} / 2 \lesssim \sigma^{1 / 2}$,

where the factor 2 accounts for the half opening-angle and the $\sigma$ is the level of jet magnetization defined by the ratio of the Poynting flux to kinetic energy (i.e., $\sigma=1$ for equipartition). For the jet viewing angle of $\theta=18^{\circ}-30^{\circ}$, the jet will be causally connected if $\Gamma \lesssim 2 \sigma^{1 / 2} / \phi_{\text {int }}=(1.3-1.8) \sigma^{1 / 2}$. If we presume $\sigma \sim 4$ at the core distance $d \sim 60 \mu$ as (jet radius $\sim 0.15$ mas; see Fig. 18 of Mertens et al. 2016), we obtain upper limits on the apparent jet speed of $\sim(2.3-3.4) c$. Previous observations, in contrast, suggest apparent speeds of $\sim 0.5 \mathrm{c}$ in this region (Hada et al. 2016; Mertens et al. 2016) and corresponding $\Gamma \phi_{\text {int }} / 2 \sim 0.7-0.9$, which satisfies Eq. (4). 
Therefore, our $\Gamma \phi_{\text {int }} / 2 \lesssim 1$ hints at a gradual collimation and acceleration of the jet base instead of a sudden acceleration accompanied by a jet breakout from a dense atmosphere, similar to GRB jet acceleration which has an order of magnitude higher $\Gamma \phi_{\text {int }} / 2$ (Panaitescu \& Kumar 2002). It is also interesting to note that $\Gamma \phi_{\text {int }} / 2 \sim 0.7-0.9$ in the M 87 jet base is significantly higher than $\Gamma \phi_{\text {int }} / 2 \sim 0.1-0.2$ found in typical pc-scale jet systems (Jorstad et al. 2005; Pushkarev et al. 2009; Clausen-Brown et al. 2013). This implies $\Gamma \phi_{\text {int }}$ is not constant for the whole AGN jet population.

It is also crucial to ascertain weather the wide expansion in the jet base can suppress different types of instabilities or not, in particular the current-driven kink instability (CDI). The CDI plays a critical role in determining the Poynting flux to kinetic energy conversion in high magnetization jet environments (e.g., Singh et al. 2016). In order for an instability to propagate across the jet, the jet expansion timescale should be longer than the timescale needed for an instability to propagate across the jet cross-section. This leads to the following criterion,

$\frac{t_{\mathrm{dyn}}}{t_{\mathrm{exp}}} \approx \frac{\beta \Gamma \phi_{\mathrm{int}} / 2}{\beta_{\mathrm{s}}} \lesssim 1$

(Komissarov et al. 2009), where $t_{\mathrm{dyn}}=\left(\Gamma \phi_{\mathrm{int}} z / 2\right) /\left(\beta_{s} c\right)$ is the jet crossing timescale for the instability, $\beta_{\mathrm{s}}$ is the signal speed in units of $c, t_{\exp }=z /(\beta c)$ is the jet expansion timescale, and $\beta$ is the speed of the jet in units of $c$. If the magnetic field is dynamically important, $\beta_{s}$ is the azimuthal component of the Alfvén speed, which is $\sim 1$ for a highly magnetized plasma (Giannios \& Spruit 2006). By using numbers corresponding to the highly magnetized M 87 jet base, we find that the condition in Eq. (5) is satisfied for the jet base. This suggests that CDIs can survive the jet expansion and could be important in terms of the dynamics and energy conversion occurring within the jet.

\subsection{Inner jet viewing angle and the outflow speed}

The viewing angle and, more importantly, the velocity of the innermost M 87 jet are crucial for our understanding of the jet geometry and dynamics. Recent GRMHD simulations show that the filamentary jet structure becomes significantly complicated near the jet formation region (e.g., Mościbrodzka et al. 2016). This can potentially make it difficult to determine precisely the viewing angle and the jet velocity. Nevertheless, previous studies (e.g., Mertens et al. 2016; Walker et al. 2016) show that the twodimensional kinematics of the jet in M 87 can be decomposed into longitudinal and transverse motions. The longitudinal motions can explain the majority of the relativistic boosting without additional jet curvature. In the light of this result, we can test two possible scenarios using the observed jet-to-counterjet ratio. The inner jet of M 87 could have (1) a stationary nonaccelerating flow, with a constant jet speed and line of sight jet orientation (i.e., constant viewing angle) or (2) the viewing angle could be constant with an accelerating jet speed.

For the case (1), if the approaching and receding jets are intrinsically of the same brightness and speed, and there is no transverse velocity gradient in the jets, the jet-to-counter-jet brightness ratio $R$ can be expressed as

$$
\begin{aligned}
& R \equiv I_{\mathrm{Jet}} / I_{\mathrm{CJ}}=\left(\frac{1+\beta \cos \theta}{1-\beta \cos \theta}\right)^{2-\alpha}, \\
& \beta=\frac{\beta_{\text {app }}}{\sin \theta+\beta_{\text {app }} \cos \theta},
\end{aligned}
$$

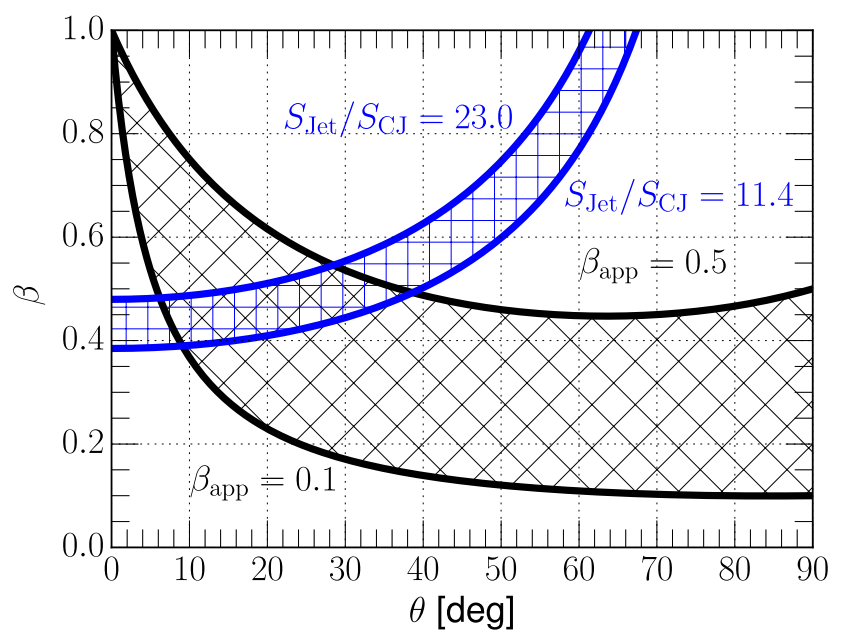

Fig. 10. Possible range of $M 87$ jet viewing angles and jet speeds (in units of $c$ ) that satisfy the measured jet-to-counter-jet ratio $R$ and our assumptions of the value of $\beta_{\text {app }}$ at the core separation of $0.2-0.5$ mas. The cross and vertical hatches show allowed ranges of the parameters for $R$ and $\beta_{\text {app }}$. Only the overlapping region is physically allowed under the assumptions outlined in Sect. 4.4.

where $I_{\mathrm{Jet}}$ and $I_{\mathrm{CJ}}$ are the intensities of the jet and the counter-jet, $\beta$ is the intrinsic jet speed normalized by the speed of light $c, \alpha$ is the spectral index $\left(S \propto v^{+\alpha}\right)$, and $\beta_{\text {app }}$ is the observed apparent speed.

It is worth noting that Eq. (6) assumes no transverse velocity gradient across the jet. A faster jet speed closer to the central axis could make the apparent jet-to-counter-jet ratio $I_{\mathrm{Jet}} / I_{\mathrm{CJ}}$ lower because of different levels of Doppler boosting in the approaching and receding flows (see Figs. 2 and 3 in Komissarov 1990). We note that kinematics studies of the inner jet of M 87 find the same intrinsic flow speeds in the boundary layers of the jet and counter-jet within observational uncertainties (Mertens et al. 2016). Hence, we do not include the transverse velocity gradient effect in the analysis of the jet-to-counter-jet ratio.

In order to find a range of $\theta$ and $\beta$ satisfying both Eqs. (6) and (7), we assume $\beta_{\text {app }} \sim 0.5$ and $\sim 0.1$ as the upper and the lower limits on the apparent speed, respectively (Hada et al. 2016; Mertens et al. 2016). This wide range represents the variety of the directly measured jet speeds. We also adopt a typical optically thin spectral index of the M 87 jet $\alpha=-1$ (Hovatta et al. 2014). The jet-to-counter-jet ratio value is taken from Sect. 3.6 $(17 \pm 6)$. In Fig. 10 we show the possible range of $\theta$ and $\beta$. The range of the possible jet viewing angle is wide $\left(6^{\circ}-38^{\circ}\right)$, mainly because of the large scatter in the apparent jet speed. If the fast speed $(\sim 0.5 \mathrm{c})$ is directly associated with the true jet flow (Mertens et al. 2016), rather large jet viewing angles of $28^{\circ}-38^{\circ}$ are expected (see Hada et al. 2016). On the other hand, recent observations found significant acceleration within the jet of M 87 (Mertens et al. 2016; Walker et al. 2016), which disfavor case (1). Therefore, we expand our analysis and put more emphasis on case (2). In this subsequent analysis we assume a viewing angle of $18^{\circ}$ (Mertens et al. 2016), which better explains fast superluminal motions in the outer jet. If the jet-tocounter-jet ratio $R$ is given as a function of the distance from the jet origin, the jet kinematic parameters including the intrinsic speed $\beta$, the apparent speed $\beta_{\text {app }}$, the bulk Lorentz factor $\Gamma$, and the Doppler factor $\delta$ can be computed along the jet axis via 


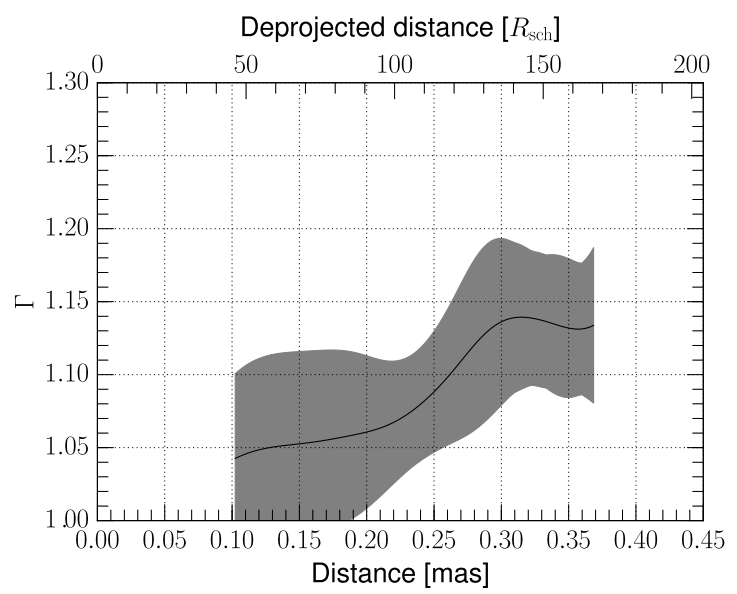

Fig. 11. Bulk Lorentz factor of the inner jet of M 87 estimated from the jet-to-counter-jet ratio vs. the distance from the central engine under the assumption of stationary viewing and changing flow speed. The solid line and the shaded region indicate the mean values and the uncertainties, respectively. The deprojected distance has been calculated based on a viewing angle of $18^{\circ}$.

$\beta=\frac{1}{\cos \theta}\left(\frac{R^{1 /(2-\alpha)}-1}{R^{1 /(2-\alpha)}+1}\right)$,

$\beta_{\text {app }}=\frac{\beta \sin \theta}{1-\beta \cos \theta}$,

$\Gamma=\frac{1}{\sqrt{1-\beta^{2}}}$.

In Fig. 11 we show the results of our calculations of the Lorenz factor $\Gamma$. In this model, the Lorentz factor mildly increases from $\sim 1.0-1.1$ at 0.1 mas to $\sim 1.1-1.2$ at $\sim 0.35$ mas, which suggests mild inner jet acceleration. In terms of the apparent speed, our fiducial model predicts $\beta_{\text {app }} \approx(0.1-0.7)$ at 0.1 mas and $(0.6-1.0)$ at $>0.3$ mas. The latter is comparable to what has been directly measured from VLBA $43 \mathrm{GHz}$ observations at similar and/or slightly larger distances (see Fig. 16 of Mertens et al. 2016). We also recall that no clear inter-day timescale structural variation was seen in the jet in the 2009 data. This implies an upper limit on the apparent jet speed of

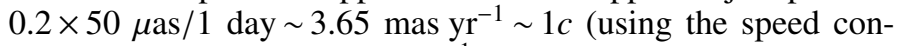

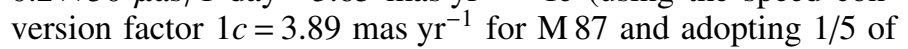
the beam size as the resolution limit). The inferred range of the apparent jet velocity agrees well with this upper limit.

We thus conclude that a mildly accelerating inner jet model with a stationary viewing angle could explain the observed inner jet-to-counter-jet ratio.

\subsection{Spine-sheath scenario in the M87 jet}

The transverse intensity profiles presented in Sect. 3.4, Figs. 4c and 5 suggest the existence of a central emission lane close to the jet base. Other deep imaging experiments also suggest similar complex structure at more distant regions along the jet (Mertens et al. 2016; Asada et al. 2016; Hada 2017; see also Sect. 4.2).

If the central lane appears fainter only due to the Doppler deboosting, then the center-to-limb brightness ratio $\rho_{C L}$ measured at the core distances $0.5-1.0$ mas can be related to the ratio of their Doppler factors by $\rho_{\mathrm{CL}}=\left(\delta_{\text {spine }} / \delta_{\text {sheath }}\right)^{2-\alpha}$. To calculate the expected range of the Spine Lorentz factor $\Gamma_{\text {spine, }}$, we assume

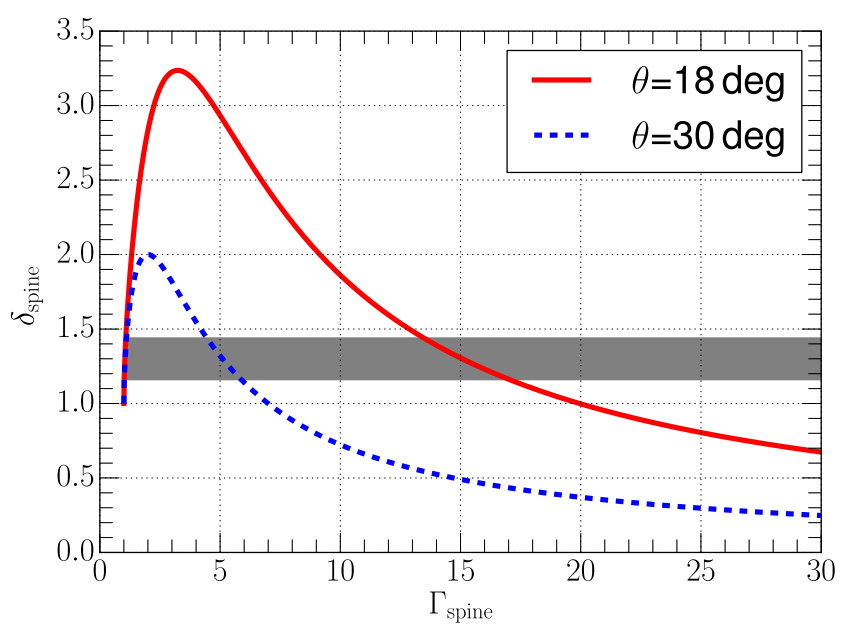

Fig. 12. Doppler factor vs. Lorentz factor for the spine. The shaded region indicates possible range of the $\delta_{\text {spine }}$ derived based on the assumption of the velocity stratification.

$\alpha=-1$, a viewing angle of $18^{\circ}-30^{\circ}$, and an apparent speed of the sheath of $\sim 0.5 \mathrm{c}$ (the corresponding $\Gamma_{\text {sheath }} \sim 1.18-1.29$ and $\left.\delta_{\text {sheath }} \sim 1.58-1.96\right)$. The observed $\rho_{\mathrm{CL}}$ constrains $\delta_{\text {spine }}$ to be $\sim 1.16-1.44$. In Fig. 12 we show the range of $\delta_{\text {spine }}$ versus the spine Lorentz factor for small and relatively large viewing angles, respectively. It is apparent from the figure that a rather fast $\Gamma_{\text {spine }} \sim 13-17\left(\Gamma_{\text {spine }} \sim 4.4-5.9\right)$ is required ${ }^{3}$ when the viewing angle is $18^{\circ}\left(30^{\circ}\right)$.

We note that the inferred $\Gamma_{\text {spine }}$ is large considering the very small distance from the core $\left(\sim 0.5-1.0\right.$ mas; $70-140 R_{\text {sch }}$ projected). For instance, $\Gamma_{\text {spine }} \sim 13-17$ is comparable to the Lorentz factor of HST-1 determined at optical wavelengths by Biretta et al. (1999) $\left(\Gamma \sim 14\right.$ for the viewing angle $\left.18^{\circ}\right)$. At such short wavelengths the observed radiation presumably traces relativistic plasma closer to the central axis of the jet (e.g., Perlman et al. 1999; Mertens et al. 2016).

General magnetohydrodynamic simulations show that accreting BH systems can form (i) a narrow, relativistic, and Poynting-dominated beam (i.e., spine) and (ii) broader, subrelativistic, and mass-dominated outflow (i.e., sheath) as a natural consequence of the mass accretion and BH physics (e.g., Hawley \& Krolik 2006; Sądowski et al. 2013). The latter helps to maintain low density levels near the central axis of the jet. In such cases, the narrow beam propagates without significant mass loading and maintains its initial speed up to large distances.

On the other hand, Asada et al. (2016) showed that the central lane and the sheath expand in a similar manner. If the collimation and acceleration pattern of the spine is qualitatively similar to that of the sheath (Asada \& Nakamura 2012; Hada et al. 2013; Asada et al. 2014; Mertens et al. 2016), the Lorentz factor of the spine may increase at larger distances. If this is the case, the smaller $\Gamma_{\text {spine }} \sim 4-6$, inferred assuming a larger inner jet viewing angle $\left(30^{\circ}\right)$, is perhaps a more plausible estimate of the intrinsic speed.

It should be noted, however, that the intrinsic synchrotron emissivity of the spine and the sheath are not necessarily the same, especially when the two different layers are launched from different origins (e.g., the spine from the $\mathrm{BH}$ while the sheath from the inner disk). Closer to the central engine, the intrinsic emissivity of the spine may decrease (Mertens et al. 2016). In addition, the central lane is comparably bright or even brighter

\footnotetext{
Here we exclude the apparent solution, i.e., $\Gamma_{\text {spine }} \sim 1$.
} 
than the limb at 1.6-5 GHz (Asada et al. 2016; see Figs. 3 and 5 therein). Such an intensity profile cannot solely be produced by a transverse velocity gradient. Therefore, the limb-brightened jet morphology close to the jet base may not be fully explained by the velocity stratification. Intrinsic differences in the jet plasma such as the jet composition and/or the magnetic field strength would need to be considered for the different jet emissivity.

\subsection{Evolution of the jet within $\lesssim 100 R_{\text {sch }}$ deprojected distances}

The commonly invoked "differential collimation" process (see Sect. 4.3.2 of Komissarov 2012) requires significant magnetic hoop stress from toroidal magnetic fields. However, this condition can be satisfied only in the "far-zone" where the toroidal field is substantially stronger than the poloidal field (e.g., Komissarov et al. 2007, 2009; Lyubarsky 2009). Therefore, a magnetic hoop stress might not be valid near the jet launching region where the poloidal field components are much more dominant (e.g., Tchekhovskoy 2015). For this scenario, we can estimate the radial distance of such a critical point from the central engine. A substantial change in the $B$-field orientation occurs when the jet radius approaches the light cylinder radius $r_{\mathrm{lc}}=c / \Omega$ (Meier 2012), where $\Omega$ is the angular speed of the outflow. Mertens et al. (2016) recently determined the light cylinder $r_{\mathrm{lc}}$ in the M 87 jet to be $\sim 20 R_{\text {sch }}$. The observed jet radius becomes comparable to $r_{\mathrm{lc}}$ at $\sim 0.2$ mas ( $28 R_{\text {sch }}$ projected) core separation. It is interesting to note that we detect a slight divergence between the data and the single power-law model at a similar core separation (see bottom panel of Fig. 6). Future imaging experiments at higher angular resolution with the EHT plus a phased Atacama Large Millimeter/submillimeter Array (ALMA; Matthews et al. 2018) will be able to reveal the structure and propagation of the M 87 jet on such spatial scales.

\section{Conclusion}

In this paper, we presented a study of the physical conditions and structure of the innermost jet in M 87 at projected distances of (7-100) $R_{\text {sch }}$ from the core. We summarize our findings and conclusions as follows:

1. Deep images of the jet base in M87 were obtained with an east-west spatial resolution of $7 R_{\text {sch }}$ using the GMVA at $86 \mathrm{GHz}$. The multi-epoch images obtained over a decade show consistency in the core-jet structure including a highly limb-brightened jet, a faint central lane between the edges of the jet, and a weak counter-jet (but no significant evidence for counter-jet limb-brightening).

2. The VLBI core has a mean resolved size of $\sim 11 R_{\text {sch }}$ at $86 \mathrm{GHz}$. The apparent brightness temperature of this compact region is $\sim(1-3) \times 10^{10} \mathrm{~K}$, nearly an order of magnitude lower than the equipartition brightness temperature $\left(\sim 2 \times 10^{11} \mathrm{~K}\right)$. This implies that the core is magnetically energy dominated. The corresponding magnetic field strength would be between $61-210 \mathrm{G}$ for an equipartition magnetic field strength of $\sim 1 \mathrm{G}$ in the VLBI core region.

3. The size of the jet launching zone is estimated assuming a self-similar jet structure. We find the diameter of the jet base of $\sim 4.0-5.5 R_{\text {sch }}$, which points to the inner accretion disk as the origin of the sheath. This size is in good agreement with the upper limit set by $86 \mathrm{GHz}$ VLBI core size measurements $\left(11 R_{\text {sch }}\right)$ and results from previous EHT observations (Doeleman et al. 2012; Akiyama et al. 2015).
4. The apparent opening angle of the jet base can be as large as $127^{\circ} \pm 22^{\circ}$ at $60 \mu$ as core separation ( $27 R_{\text {sch }}$ deprojected). Combining the jet geometry and the kinematic information, we find that the jet base is still causally connected despite the unusually wide opening angle $\left(\Gamma \phi_{\text {int }} / 2=0.7-0.9\right)$. On the other hand, a timescale analysis shows that the jet base region can be sensitive to the current driven instabilities in the strong magnetic field regime.

5. We considered two distinct jet models, one in which the viewing angle and the speed of the jet was fixed and the other in which the viewing angle was fixed but the jet speed varied with distance from the central engine. We find jet speeds ranging from $0.1-0.5 c$ for the former and $0.1-1.0 c$ for the latter.

6. We investigated the origin of the faint central emission lane at $86 \mathrm{GHz}$ by first considering a pure transverse velocity stratification scenario (i.e., the spine-sheath model). We constrained the Lorentz factor of the spine to be $\Gamma_{\text {spine }} \sim 13-17$ (4-6) for $\theta=18^{\circ}\left(30^{\circ}\right)$ and compared it with the Lorentz factor of HST-1 determined at optical wavelengths $(\Gamma \sim 14)$. These estimates are consistent with a nearly constant velocity of the spine $\left(\theta=18^{\circ}\right)$ or an accelerating spine with a rather large inner jet viewing angle $\left(\theta=30^{\circ}\right)$. However, we notice that the relative brightness of the central lane with respect to the edges increases significantly at longer centimeter wavelengths. Therefore, we suggest that the edge-brightening in the jet of M 87 may not be driven only by the velocity gradient but also by intrinsic differences (i.e., composition, magnetic field strength) in the plasma within the lane and the sheath.

Acknowledgements. We sincerely thank the referee, Serguei S. Komissarov, for the review and comments which improved the interpretation of our results. We also thank Nicholas R. MacDonald for the careful reading and helpful comments. J.-Y. Kim is supported for this research by the International Max-Planck Research School (IMPRS) for Astronomy and Astrophysics at the University of Bonn and Cologne. E.R. was partially supported by the Spanish MINECO grant AYA2015-63939-C2-2-P and by the Generalitat Valenciana grant PROMETEOII/2014/057. This research has made use of data obtained with the Global Millimeter VLBI Array (GMVA), which consists of telescopes operated by the MPIfR, IRAM, Onsala, Metsahovi, Yebes, the Korean VLBI Network, the Green Bank Observatory, and the Long Baseline Observatory (LBO). The VLBA is an instrument of the LBO, which is a facility of the National Science Foundation operated by Associated Universities, Inc. The data were correlated at the correlator of the MPIfR in Bonn, Germany. This work made use of the Swinburne University of Technology software correlator, developed as part of the Australian Major National Research Facilities Programme and operated under licence.

\section{References}

Abramowski, A., Acero, F., Aharonian, F., et al. 2012, ApJ, 746, 151

Acciari, V. A., Aliu, E., Arlen, T., et al. 2010, ApJ, 716, 819

Akiyama, K., Lu, R.-S., Fish, V. L., et al. 2015, ApJ, 807, 150

Asada, K., \& Nakamura, M. 2012, ApJ, 745, L28

Asada, K., Nakamura, M., Doi, A., Nagai, H., \& Inoue, M. 2014, ApJ, 781, L2

Asada, K., Nakamura, M., \& Pu, H.-Y. 2016, ApJ, 833, 56

Baczko, A.-K., Schulz, R., Kadler, M., et al. 2016, A\&A, 593, A47

Bird, S., Harris, W. E., Blakeslee, J. P., \& Flynn, C. 2010, A\&A, 524, A71

Biretta, J. A., Sparks, W. B., \& Macchetto, F. 1999, ApJ, 520, 621

Blandford, R. D., \& Payne, D. G. 1982, MNRAS, 199, 883

Blandford, R. D., \& Znajek, R. L. 1977, MNRAS, 179, 433

Boccardi, B., Krichbaum, T. P., Bach, U., Bremer, M., \& Zensus, J. A. 2016a, A\&A, 588, L9

Boccardi, B., Krichbaum, T. P., Bach, U., et al. 2016b,A\&A, 585, A33

Broderick, A. E., \& Loeb, A. 2009, ApJ, 697, 1164

Carilli, C. L., Bartel, N., \& Linfield, R. P. 1991, AJ, 102, 1691

Cheung, C. C., Harris, D. E., \& Stawarz, Ł. 2007, ApJ, 663, L65

Clausen-Brown, E., Savolainen, T., Pushkarev, A. B., Kovalev, Y. Y., \& Zensus, J. A. 2013, A\&A, 558, A144

Deller, A. T., Tingay, S. J., Bailes, M., \& West, C. 2007, PASP, 119, 318 
Doeleman, S. S., Fish, V. L., Schenck, D. E., et al. 2012, Science, 338, 355

Fromm, C. M., Ros, E., Perucho, M., et al. 2013, A\&A, 557, A105

Gebhardt, K., Adams, J., Richstone, D., et al. 2011, ApJ, 729, 119

Ghisellini, G., Guilbert, P. W., \& Svensson, R. 1988, ApJ, 334, L5

Giannios, D., \& Spruit, H. C. 2006, A\&A, 450, 887

Giovannini, G., Savolainen, T., Orienti, M., et al. 2018, NatAs., 2, 472

Greisen, E. W. 1990, in Acquisition, Processing and Archiving of Astronomica Images, eds. G. Longo, \& G. Sedmak, (Napoli: Finito di stampare da Officine Grafiche Liguori), 125

Hada, K. 2017, Galaxies, 5, 2

Hada, K., Doi, A., Kino, M., et al. 2011, Nature, 477, 185

Hada, K., Kino, M., Doi, A., et al. 2013, ApJ, 775, 70

Hada, K., Giroletti, M., Kino, M., et al. 2014, ApJ, 788, 165

Hada, K., Kino, M., Doi, A., et al. 2016, ApJ, 817, 131

Hardee, P., Mizuno, Y., \& Nishikawa, K.-I. 2007, Ap\&SS, 311, 281

Hawley, J. F., \& Krolik, J. H. 2006, ApJ, 641, 103

Högbom, J. A. 1974, A\&AS, 15, 417

Hovatta, T., Aller, M. F., Aller, H. D., et al. 2014, AJ, 147, 143

Jorstad, S. G., Marscher, A. P., Lister, M. L., et al. 2005, AJ, 130, 1418

Junor, W., Biretta, J. A., \& Livio, M. 1999, Nature, 401, 891

Kellermann, K. I., \& Pauliny-Toth, I. I. K. 1969, ApJ, 155, L71

Kim, J.-Y., Lu, R.-S., Krichbaum, T., et al. 2016, Galaxies, 4, 39

Kim, J.-Y., Lee, S.-S., Hodgson, J. A., et al. 2018, A\&A, 610

Kino, M., Takahara, F., Hada, K., et al. 2015, ApJ, 803, 30, L5

Komatsu, E., Smith, K. M., Dunkley, J., et al. 2011, ApJS, 192, 18

Komissarov, S. S. 1990, Sov. Astron. Lett., 16, 284

Komissarov, S. 2012, in Central Engines: Acceleration, Collimation and Confinement of Jets, eds. M. Boettcher, D. E. Harris, \& H. Krawczynski, (Weinheim: WILEY-VCH Verlag), 81

Komissarov, S. S., Barkov, M. V., Vlahakis, N., \& Königl, A. 2007, MNRAS, 380,51

Komissarov, S. S., Vlahakis, N., Königl, A., \& Barkov, M. V. 2009, MNRAS, 394, 1182

Komissarov, S. S., Vlahakis, N., \& Königl, A. 2010, MNRAS, 407, 17

Kovalev, Y. Y., Kellermann, K. I., Lister, M. L., et al. 2005, AJ, 130, 2473

Kovalev, Y. Y., Lister, M. L., Homan, D. C., \& Kellermann, K. I. 2007, ApJ, 668 L27

Krichbaum, T. P., Agudo, I., Bach, U., Witzel, A., \& Zensus, J. A. 2006, in Proceedings of the 8th European VLBI Network Symposium, 2

Krichbaum, T. P., Roy, A., Lu, R.-S., et al. 2014, in Proceedings of the 12th European VLBI Network Symposium and Users Meeting (EVN 2014), October 2014, Cagliari, Italy, 13

Lee, S.-S., Lobanov, A. P., Krichbaum, T. P., \& Zensus, J. A. 2016, ApJ, 826, 135

Lobanov, A. P. 1998, A\&A, 330, 79

Lobanov, A. P. 2005, ArXiv e-prints [arXiv:astro-ph/0503225]

Ly, C., Walker, R. C., \& Junor, W. 2007, ApJ, 660, 200
Lyubarsky, Y. 2009, ApJ, 698, 1570

MacDonald, N. R., Marscher, A. P., Jorstad, S. G., \& Joshi, M. 2015, ApJ, 804, 111

Marscher, A. P. 1983, ApJ, 264, 296

Martí-Vidal, I., Krichbaum, T. P., Marscher, A., et al. 2012, A\&A, 542, A107

Martí-Vidal, I., Muller, S., Vlemmings, W., Horellou, C., \& Aalto, S. 2015, Science, 348,311

Matthews, L. D., Crew, G. B., Doeleman, S. S., et al. 2018, PASP, 130, 015002

McKinney, J. C. 2006, MNRAS, 368, 1561

Meier, D. L. 2012, in Black Hole Astrophysics: The Engine Paradigm (Berlin Heidelberg: Springer-Verlag)

Mertens, F., Lobanov, A. P., Walker, R. C., \& Hardee, P. E. 2016, A\&A, 595, A54

Mościbrodzka, M., Falcke, H., \& Shiokawa, H. 2016, A\&A, 586, A38

Nair, D. G., Lobanov, A. P., Krichbaum, T. P., et al. 2018, A\&A, accepted

Panaitescu, A., \& Kumar, P. 2002, ApJ, 571, 779

Perlman, E. S., Biretta, J. A., Zhou, F., Sparks, W. B., \& Macchetto, F. D. 1999, AJ, 117, 2185

Prieto, M. A., Fernández-Ontiveros, J. A., Markoff, S., Espada, D., \& GonzálezMartín, O. 2016, MNRAS, 457, 3801

Pu, H.-Y., Wu, K., Younsi, Z., et al. 2017, ApJ, 845, 160

Pushkarev, A. B., Kovalev, Y. Y., Lister, M. L., \& Savolainen, T. 2009, A\&A, 507, L33

Pushkarev, A. B., Hovatta, T., Kovalev, Y. Y., et al. 2012, A\&A, 545, A113

Pushkarev, A. B., Kovalev, Y. Y., Lister, M. L., \& Savolainen, T. 2017, MNRAS, 468, 4992

Readhead, A. C. S. 1994, ApJ, 426, 51

Sạdowski, A., Narayan, R., Penna, R., \& Zhu, Y. 2013, MNRAS, 436, 3856

Schinzel, F. K., Lobanov, A. P., Taylor, G. B., et al. 2012, A\&A, 537, A70

Shepherd, M. C., Pearson, T. J., \& Taylor, G. B. 1994, BAAS, 26, 987

Singal, A. K. 2009, ApJ, 703, L109

Singh, C. B., Mizuno, Y., \& de Gouveia Dal Pino, E. M. 2016, ApJ, 824, 48

Smith, R. J., Lucey, J. R., Hudson, M. J., Schlegel, D. J., \& Davies, R. L. 2000, MNRAS, 313, 469

Tchekhovskoy, A. 2015, in The Formation and Disruption of Black Hole Jets, eds. I. Contopoulos, D. Gabuzda, \& N. Kylafis, Astrophys. Space Sci. Lib., 414, 45

Tchekhovskoy, A., Narayan, R., \& McKinney, J. C. 2010, New Ast., 15, 749

Tchekhovskoy, A., Narayan, R., \& McKinney, J. C. 2011, MNRAS, 418, L79

Walker, R. C., Ly, C., Junor, W., \& Hardee, P. J. 2008, J. Phys. Conf. Ser., 131, 012053

Walker, R. C., Hardee, P. E., Davies, F., et al. 2016, Galaxies, 4, 46

Walsh, J. L., Barth, A. J., Ho, L. C., \& Sarzi, M. 2013, ApJ, 770, 86

Yuan, F., \& Narayan, R. 2014, ARA\&A, 52, 529

Zamaninasab, M., Clausen-Brown, E., Savolainen, T., \& Tchekhovskoy, A. 2014, Nature, 510, 126 